\title{
Two sequential fractional hybrid differential inclusions
}

\author{
Hakimeh Mohammadi ${ }^{1}$, Shahram Rezapour ${ }^{2,3,4^{*}}$ (D), Sina Etemad ${ }^{5}$ and Dumitru Baleanu ${ }^{6,7}$
}

\author{
"Correspondence: \\ shahramrezapour@duytan.edu.vn; \\ sh.rezapour@mail.cmuh.org.tw; \\ sh.rezapour@azaruniv.ac.ir; \\ rezapourshahram@yahoo.ca \\ ${ }^{2}$ Institute of Research and \\ Development, Duy Tan University, \\ Da Nang 550000, Vietnam \\ ${ }^{3}$ Faculty of Natural Sciences, Duy \\ Tan University, Da Nang 550000, \\ Vietnam \\ Full list of author information is \\ available at the end of the article
}

\begin{abstract}
The main objective of this paper is to concern with a new category of the sequential hybrid inclusion boundary value problem with three-point integro-derivative boundary conditions. In this direction, we employ various novel analytical techniques based on $\alpha$ - $\psi$-contractive mappings, endpoints, and the fixed points of the product operators to obtain the main results. Finally, we provide two examples to illustrate our main results.
\end{abstract}

MSC: Primary 34A08; secondary 34A12

Keywords: $\alpha-\psi$-contraction; Endpoint; Sequential hybrid inclusion problem; The Caputo derivative

\section{Introduction}

Every day that passes, the human needs for studying natural phenomena is increasing. One of the possible methods for achieving this goal is using mathematical operators and computer modeling. The fractional operators were developed over the years, and their importance has become more and more apparent to researchers today. Instances of the application of such fractional operators can be found in various sciences such as biomathematics, electrical circuits, medicine, and so on [1-6]. All these items have led researchers to find many aspects of the structure of the fractional boundary value problems and hereditary properties of their solutions. In this regard, many researchers investigated advanced fractional modelings $[7,8]$ and related theoretical results and qualitative behaviors of such fractional boundary value problems [9-22].

There have been appeared different versions of fractional operators during these years. In monograph [23], Miller and Ross defined another type of fractional derivative called sequential derivatives, which ares a combination of the existing derivative operators. Later, the attention of some researchers was attracted to finding a connection between the usual Riemann-Liouville derivative and the sequential fractional derivative $[24,25]$. These useful results have led to publishing some papers on the sequential fractional boundary value problems (see, e.g., [26-30]). In 2015, Alsaedi et al. studied the sequential problem

$$
\left\{\begin{array}{l}
\left({ }^{c} D_{0^{+}}^{\alpha^{*}}+k^{c} D_{0^{+}}^{\alpha^{*}-1}\right) w(s)=g(s, w(s)), \quad s \in[0,1], \\
w(0)=0, \quad w^{\prime}(0)=0, \quad w(\xi)=a^{R} I_{0^{+}}^{\beta} w(\eta),
\end{array}\right.
$$

(c) The Author(s) 2020. This article is licensed under a Creative Commons Attribution 4.0 International License, which permits use, sharing, adaptation, distribution and reproduction in any medium or format, as long as you give appropriate credit to the original author(s) and the source, provide a link to the Creative Commons licence, and indicate if changes were made. The images or other third party material in this article are included in the article's Creative Commons licence, unless indicated otherwise in a credit line to the material. If material is not included in the article's Creative Commons licence and your intended use is not permitted by statutory regulation or exceeds the permitted use, you will need to obtain permission directly from the copyright holder. To view a copy of this licence, visit http://creativecommons.org/licenses/by/4.0/. 
where $\alpha^{*} \in(2,3], \xi \in(\eta, 1)$, with $\eta, \beta>0, a, k \in \mathbb{R}^{+}$, and $g:[0,1] \times \mathbb{R} \rightarrow \mathbb{R}$ is a continuous function [31]. On the other hand, hybrid differential problems with different kinds of boundary conditions have gained extensive attention of many researchers [32-34]. This area begins with a joint work presented by Dhage and Lakshmikantham [35] in 2010. The authors addressed a novel differential equation entitled a hybrid differential equation and investigated the extremal solutions of this new BVP by deriving some useful fundamental differential inequalities [35]. Later, Zhao et al. [36] gave an abstract extension for the problem mentioned in [35] to fractional order and defined a boundary value problem of fractional hybrid differential equations. Until now, the limited research papers have been published on various properties of solutions for hybrid boundary value problems of fractional order. In 2016, Ahmad et al. [37]performed an important existence analysis for the nonlocal fractional BVP of hybrid inclusion problem given by

$$
{ }^{c} D_{0^{+}}^{\varrho}\left[\frac{w(s)-\sum_{j=1}^{k}{ }^{R} I_{0^{+}}^{\varpi_{j}} h_{j}(s, w(s))}{g(s, w(s))}\right] \in \mathcal{G}(s, w(s)), \quad s \in \mathcal{I}=[0,1],
$$

with boundary conditions $w(0)=\beta(s)$ and $w(1)=\alpha \in \mathbb{R}$, where ${ }^{c} D_{0^{+}}^{\varrho}$ is the Caputo derivative of order $\varrho \in(1,2]$, and ${ }^{R} I_{0^{+}}^{\varphi}$ is the Riemann-Liouville integral of order $\varphi>0$ such that $\varphi \in\left\{\beta_{1}, \beta_{2}, \ldots, \beta_{m}\right\}$. After that, Baleanu et al. [38] developed some existence theorems and arrived at some findings on the dimension of the set of all solutions for the hybrid inclusion problem

$$
{ }^{c} D_{0^{+}}^{\varrho}\left[\frac{w(s)}{\Lambda\left(s, w(s),{ }^{R} I_{0^{+}}^{\omega_{1}} w(s), \ldots,{ }^{R} I_{0^{+}}^{\omega_{n}} w(s)\right)}\right] \in \mathcal{G}\left(s, w(s),{ }^{R} I_{0^{+}}^{\alpha_{1}} w(s), \ldots,{ }^{R} I_{0^{+}}^{\alpha_{m}} w(s)\right)
$$

with boundary conditions $w(0)=w_{0}^{*}$ and $w(1)=w_{1}^{*}$, where $s \in[0,1], \varrho \in(1,2],{ }^{c} D_{0^{+}}^{\varrho}$ and ${ }^{R} I_{0^{+}}^{\gamma}$ denote the Caputo derivative operator of order $\varrho$ and the Riemann-Liouville integral operator of order $\gamma \in\left\{\varpi_{i}, \alpha_{j}\right\} \subset(0, \infty)$ for $i=1, \ldots n$ and $j=1, \ldots, m$, respectively. In 2019, Baleanu et al. [39] introduced a new hybrid problem in which, for the first time, the boundary conditions were considered as hybrid type. Indeed, the authors formulated the following fractional three-point hybrid problem:

$$
{ }^{c} D_{0^{+}}^{\varrho}\left[\frac{w(s)}{\rho(s, w(s))}\right]=\kappa(s, w(s)), \quad s \in[0,1]
$$

with three-point mixed integral hybrid conditions

$$
\left\{\begin{array}{l}
w(0)=0, \\
{\left.\left[\frac{w(s)}{\rho(s, w(s))}\right]\right|_{s=0}+\left.{ }^{R} I_{o^{+}}^{e^{*}}\left[\frac{w(s)}{\rho(s, w(s))}\right]\right|_{s=\eta}=0,} \\
{\left.\left[\frac{w(s)}{\rho(s, w(s))}\right]\right|_{s=0}+\left.{ }^{R} I_{0^{+}}^{e^{*}}\left[\frac{w(s)}{\rho(s, w(s))}\right]\right|_{s=1}=0,}
\end{array}\right.
$$

where $\varrho \in(2,3], \varrho^{*}>0$, and $\eta \in(0,1)$. The function $\kappa:[0,1] \times \mathbb{R} \rightarrow \mathbb{R}$ is continuous, and $\rho \in \mathcal{C}([0,1] \times \mathbb{R}, \mathbb{R} \backslash\{0\})$. In 2020, Baleanu et al. [7] designed a new fractional hybrid model of thermostat in which the thermostat controls an amount of heat based on the temperature detected by sensors. This hybrid model is illustrated by

$$
{ }^{c} D_{0^{+}}^{\varrho}\left[\frac{w(s)}{h(s, w(s))}\right]+\Phi(s, w(s))=0, \quad \varrho \in(1,2], s \in[0,1],
$$


with fractional hybrid boundary value conditions

$$
\left\{\begin{array}{l}
\left.D\left[\frac{w(s)}{h(s, w(s))}\right]\right|_{s=0}=0, \\
\left.\lambda^{c} D_{0^{+}}^{\varrho-1}\left[\frac{w(s)}{h(s, w(s))}\right]\right|_{s=1}+\left.\left[\frac{w(s)}{h(s, w(s))}\right]\right|_{s=\eta}=0,
\end{array}\right.
$$

where $\lambda>0$ is a parameter, $\eta \in[0,1]$, and $\varrho-1 \in(0,1]$. Also, $D=\frac{\mathrm{d}}{\mathrm{d} s},{ }^{c} D_{0^{+}}^{\gamma}$ is the Caputo derivative of fractional order $\gamma \in\{\varrho, \varrho-1\}$, the function $\Phi:[0,1] \times \mathbb{R} \rightarrow \mathbb{R}$ is continuous, and $h \in \mathcal{C}([0,1] \times \mathbb{R}, \mathbb{R} \backslash\{0\})$.

Now by combining ideas of these works, we firstly turn to the sequential fractional hybrid differential inclusion of Caputo type

$$
p_{1}\left({ }^{c} D_{0^{+}}^{\varrho}+p_{2}{ }^{c} D_{0^{+}}^{\varrho-1}\right)\left[\frac{w(s)}{\zeta\left(s, w(s),{ }^{R} I_{0^{+}}^{\gamma} w(s)\right)}\right] \in \mathcal{S}(s, w(s)), \quad s \in[0,1],
$$

with three-point hybrid integro-derivative boundary conditions

$$
\left\{\begin{array}{l}
{\left.\left[\frac{w(s)}{\zeta\left(s, w(s),{ }^{R} I_{0^{+}}^{\gamma} w(s)\right)}\right]\right|_{s=0}=0,} \\
\left.{ }^{c} D_{0^{+}}^{1}\left[\frac{w(s)}{\zeta\left(s, w(s),{ }^{R} I_{0^{+}}^{\gamma} w(s)\right.}\right]\right|_{s=0}+\left.{ }^{c} D_{0^{+}}^{2}\left[\frac{w(s)}{\zeta\left(s, w(s),{ }^{R} I_{0^{+}}^{\gamma} w(s)\right)}\right]\right|_{s=0}=0, \\
{\left.\left[\frac{w(s)}{\zeta\left(s, w(s),{ }^{\prime} I_{0^{+}}^{\gamma} w(s)\right)}\right]\right|_{s=1}+\left.{ }^{R} I_{0^{+}}^{\xi}\left[\frac{w(s)}{\zeta\left(s, w(s),{ }^{\prime} I_{0^{+}}^{\gamma} w(s)\right)}\right]\right|_{s=p}=0,}
\end{array}\right.
$$

where $\varrho \in(2,3], p \in(0,1), p_{1}, p_{2}, \gamma, \xi>0,{ }^{c} D_{0^{+}}^{(\cdot)}$ and ${ }^{R} I_{0^{+}}^{(\cdot)}$ denote the Caputo fractional derivative and the Riemann-Liouville fractional integral, respectively. Note that ${ }^{c} D_{0^{+}}^{1}=\frac{\mathrm{d}}{\mathrm{d} s}$ and ${ }^{c} D_{0^{+}}^{2}=\frac{\mathrm{d}^{2}}{\mathrm{ds} s^{2}}$. The nonzero continuous real-valued function $\zeta$ is supposed to be defined on $[0,1] \times \mathbb{R} \times \mathbb{R}$, and $\mathcal{S}:[0,1] \times \mathbb{R} \rightarrow \mathcal{P}(\mathbb{R})$ is a set-valued map equipped via some properties. Also, we investigate the special case of the sequential hybrid inclusion BVP

$$
p_{1}\left({ }^{c} D_{0^{+}}^{\varrho}+p_{2}{ }^{c} D_{0^{+}}^{Q-1}\right) w(s) \in \mathcal{S}(s, w(s)), \quad s \in[0,1],
$$

with three-point integro-derivative boundary conditions

$$
w(0)=0, \quad w^{\prime}(0)+w^{\prime \prime}(0)=0, \quad w(1)+{ }^{R} I_{0^{+}}^{\xi} w(p)=0,
$$

where $\varrho \in(2,3], p \in(0,1), p_{1}, p_{2}>0$, and ${ }^{R} I_{0^{+}}^{\xi}$ denotes the Riemann-Liouville fractional integral of order $\xi>0$. It is obvious that the sequential inclusion problem (3)-(4) is derived whenever $\zeta\left(s, w(s),{ }^{R} I_{0^{+}}^{\gamma} w(s)\right)=1$.

\section{Preliminaries}

Let $\varrho>0$. The definition of the Riemann-Liouville integral of a function $w:[0,+\infty) \rightarrow \mathbb{R}$ is of the form of ${ }^{R} I_{0^{+}}^{\varrho} w(s)=\int_{0}^{s} \frac{(s-r)^{\varrho-1}}{\Gamma(\varrho)} w(r) \mathrm{d} r$, provided that the value of the integral is finite $[40,41]$. Let $\varrho \in(n-1, n)$ be such that $n=[\varrho]+1$. For a function $w \in \mathcal{A C}_{\mathbb{R}}^{(n)}([0,+\infty))$, the fractional derivative of Caputo type is given by ${ }^{c} D_{0^{+}}^{\varrho} w(s)=\int_{0}^{s} \frac{(s-r)^{n-\varrho-1}}{\Gamma(n-\varrho)} w^{(n)}(r) \mathrm{d} r$, provided that the integral is finite-valued [40,41]. Moreover, for a sufficiently smooth function $w$ : $[0,+\infty) \rightarrow \mathbb{R}$, the sequential fractional derivative is defined by

$$
D_{0^{+}}^{\varrho} w(s)=\left(D_{0^{+}}^{\varrho_{1}} D_{0^{+}}^{\varrho_{2}} \ldots D_{0^{+}}^{\varrho_{n}}\right) w(s),
$$


where $\varrho=\left(\varrho_{1}, \varrho_{2}, \ldots, \varrho_{n}\right)$ is a multiindex [23]. Note that, in general, the sequential derivative operator $D_{0^{+}}^{\varrho}$ can be Riemann-Liouville, Caputo, Hadamard, Caputo-Hadamard, or any other version of derivative operators. In this research, we employ the sequential derivative of Caputo type defined as follows. For $n-1<\varrho<n$, the Caputo sequential fractional derivative for a sufficiently smooth function $w:[0,+\infty) \rightarrow \mathbb{R}$ is given by

$$
{ }^{c} D_{0^{+}}^{\varrho} w(s)=D_{0^{+}}^{-(n-\varrho)}\left(\frac{\mathrm{d}}{\mathrm{d} s}\right)^{n} w(s)
$$

where $D_{0^{+}}^{-(n-\varrho)} w(s)={ }^{R} I_{0^{+}}^{(n-\varrho)} w(s)$ is the Riemann-Liouville fractional integral of order $n-\varrho$ [40]. It has been verified that the general solution for the homogeneous differential equation ${ }^{c} D_{0^{+}}^{\varrho} w(s)=0$ is given by $w(s)=\tilde{m}_{0}+\tilde{m}_{1} s+\tilde{m}_{2} s^{2}+\cdots+\tilde{m}_{n-1} s^{n-1}$ and

$$
{ }^{R} I_{0^{+}}^{\varrho}\left({ }^{c} D_{0^{+}}^{\varrho} w(s)\right)=w(s)+\sum_{k=0}^{n-1} \tilde{m}_{k} s^{k}=w(s)+\tilde{m}_{0}+\tilde{m}_{1} s+\tilde{m}_{2} s^{2}+\cdots+\tilde{m}_{n-1} s^{n-1},
$$

where $\tilde{m}_{0}, \ldots, \tilde{m}_{n-1}$ are real numbers with $n=[\varrho]+1$ [23]. Here we recall some important and required properties in the Banach spaces. For this purpose, consider the normed space $\left(\mathcal{W},\|\cdot\|_{\mathcal{W}}\right)$. For convenience, we denote by $\mathcal{P}(\mathcal{W}), \mathcal{P}_{\text {cls }}(\mathcal{W}), \mathcal{P}_{\text {bnd }}(\mathcal{W}), \mathcal{P}_{\text {cmp }}(\mathcal{W})$, and $\mathcal{P}_{\text {cvx }}(\mathcal{W})$ the collections of all subsets, all closed subsets, all bounded subsets, all compact subsets, and all convex subsets of $\mathcal{W}$, respectively. The element $w^{*} \in \mathcal{W}$ is a fixed point for a given set-valued map $\mathcal{S}: \mathcal{W} \rightarrow \mathcal{P}(\mathcal{W})$ whenever $w^{*} \in \mathcal{S}\left(w^{*}\right)$ [42]. We denote the family of all fixed points of $\mathcal{S}$ by $\mathcal{F} \mathcal{I} \mathcal{X}(\mathcal{S})$ [42]. The Pompeiu-Hausdorff metric $\mathrm{PH}_{d_{\mathcal{W}}}: \mathcal{P}(\mathcal{W}) \times \mathcal{P}(\mathcal{W}) \rightarrow \mathbb{R}^{*}=\mathbb{R} \cup\{\infty\}$ is defined by

$$
\operatorname{PH}_{d_{\mathcal{W}}}\left(A_{1}, A_{2}\right)=\max \left\{\sup _{a_{1} \in A_{1}} d_{\mathcal{W}}\left(a_{1}, A_{2}\right), \sup _{a_{2} \in A_{2}} d_{\mathcal{W}}\left(A_{1}, a_{2}\right)\right\}
$$

where $d_{\mathcal{W}}\left(A_{1}, a_{2}\right)=\inf _{a_{1} \in A_{1}} d_{\mathcal{W}}\left(a_{1}, a_{2}\right)$ and $d_{\mathcal{W}}\left(a_{1}, A_{2}\right)=\inf _{a_{2} \in A_{2}} d_{\mathcal{W}}\left(a_{1}, a_{2}\right)$ [42]. A setvalued map $\mathcal{S}: \mathcal{W} \rightarrow \mathcal{P}_{\text {cls }}(\mathcal{W})$ is Lipschitzian with positive constant $\hat{\lambda}$ if $\operatorname{PH}_{d_{\mathcal{W}}}(\mathcal{S}(w)$, $\left.\mathcal{S}\left(w^{\prime}\right)\right) \leq \hat{\lambda} d_{\mathcal{W}}\left(w, w^{\prime}\right)$ for all $w, w^{\prime} \in \mathcal{W}$. A Lipschitz map $\mathcal{S}$ is a contraction if $\hat{\lambda} \in(0,1)$ [42]. A map $\mathcal{S}$ is said to be completely continuous if $\mathcal{S}(K)$ is relatively compact for each $K \in \mathcal{P}_{\text {bnd }}(\mathcal{W})$, whereas $\mathcal{S}:[0,1] \rightarrow \mathcal{P}_{\text {cls }}(\mathbb{R})$ is called measurable if $s \longmapsto d_{\mathcal{W}}(v, \mathcal{S}(s))$ is measurable for any $v \in \mathbb{R}[42,43]$. Also, $\mathcal{S}$ is an upper semicontinuous if for every $w^{*} \in \mathcal{W}$, the set $\mathcal{S}\left(w^{*}\right)$ belongs to $\mathcal{P}_{\text {cls }}(\mathcal{W})$ and for each open set $\mathcal{U}$ containing $\mathcal{S}\left(w^{*}\right)$, there is a neighborhood $\mathcal{O}_{0}^{*}$ of $w^{*}$ such that that $\mathcal{S}\left(\mathcal{O}_{0}^{*}\right) \subseteq \mathcal{U}[42]$. We construct the graph of the set-valued $\operatorname{map} \mathcal{S}: \mathcal{W} \rightarrow \mathcal{P}_{\text {cls }}(\mathcal{Z})$ by $\operatorname{Graph}(\mathcal{S})=\{(w, z) \in \mathcal{W} \times \mathcal{Z}: z \in \mathcal{S}(w)\}$. The $\operatorname{Graph}(\mathcal{S})$ is closed if for two arbitrary convergent sequences $\left\{w_{n}\right\}_{n \geq 1}$ in $\mathcal{W}$ and $\left\{z_{n}\right\}_{n \geq 1}$ in $\mathcal{Z}$ with $w_{n} \rightarrow w_{0}$, $z_{n} \rightarrow z_{0}$, and $z_{n} \in \mathcal{S}\left(w_{n}\right)$, we have the inclusion $z_{0} \in \mathcal{S}\left(w_{0}\right)$ [42, 43]. In view of [42], it is deduced that if the set-valued map $\mathcal{S}: \mathcal{W} \rightarrow \mathcal{P}_{\text {cls }}(\mathcal{Z})$ is upper semicontinuous, then $\operatorname{Graph}(\mathcal{S})$ is a closed subset of $\mathcal{W} \times \mathcal{Z}$. On the contrary, if $\mathcal{S}$ is completely continuous and closed (i.e., has a closed graph), then $\mathcal{S}$ is upper semicontinuous [42]. In addition, $\mathcal{S}$ has convex values if $\mathcal{S}(k) \in \mathcal{P}_{\text {cvx }}(\mathcal{W})$ for each $w \in \mathcal{W}$. Furthermore, a collection of selections of $\mathcal{S}$ at point $w \in \mathcal{C}_{\mathbb{R}}([0,1])$ is represented by $(\mathcal{S E L})_{\mathcal{S}, w}:=\left\{\hat{\vartheta} \in \mathcal{L}_{\mathbb{R}}^{1}([0,1]): \hat{\vartheta}(s) \in \mathcal{S}(s, w(s))\right\}$ for (a.e.) $s \in[0,1][42,43]$. Note that if $\mathcal{S}$ is an arbitrary set-valued map, then for each $w \in \mathcal{C}_{\mathcal{W}}([0,1])$, we have $(\mathcal{S E L})_{\mathcal{S}, w} \neq \emptyset$ whenever $\operatorname{dim}(\mathcal{W})<\infty[42]$. We say that $\mathcal{S}:[0,1] \times \mathbb{R} \rightarrow \mathcal{P}(\mathbb{R})$ is Carathéodory if $s \mapsto \mathcal{S}(s, w)$ is measurable for every $w \in \mathbb{R}$ and $w \mapsto \mathcal{S}(s, w)$ is upper 
semicontinuous for (a.e.) $w \in[0,1]([42,43])$. Besides, a Carathéodory set-valued map $\mathcal{S}:[0,1] \times \mathbb{R} \rightarrow \mathcal{P}(\mathbb{R})$ is called $\mathcal{L}^{1}$-Carathéodory if for each $\mu>0$, there is $\varphi_{\mu} \in \mathcal{L}_{\mathbb{R}^{+}}^{1}([0,1])$ such that $\|\mathcal{S}(s, w)\|=\sup _{s \in[0,1]}\{|q|: q \in \mathcal{S}(s, w)\} \leq \varphi_{\mu}(s)$ for all $|w| \leq \mu$ and for almost every $s \in[0,1][42,43]$.

Samet et al. [44] introduced a new collection of nondecreasing nonnegative functions $\psi:[0, \infty) \rightarrow[0, \infty)$ with $\sum_{n=1}^{\infty} \psi^{n}(s)<\infty$, which is represented by $\Psi$. By considering the properties of these functions it is obvious that $\psi(s)<s$ for all $s>0$ [44]. Later, Mohammadi et al. [45] constructed a new structure for set-valued maps with the following definition. A set-valued map $\mathcal{S}: \mathcal{W} \rightarrow \mathcal{P}_{\text {cls,bnd }}(\mathcal{W})$ is said to be $\alpha$ - $\psi$-contraction if

$$
\alpha\left(w, w^{\prime}\right) \operatorname{PH}_{d_{\mathcal{W}}}\left(\mathcal{S} w, \mathcal{S}^{\prime}\right) \leq \psi\left(d_{\mathcal{W}}\left(w, w^{\prime}\right)\right)
$$

for all $w, w^{\prime} \in \mathcal{W}$ [45]. In addition, we say that $\mathcal{W}$ has property $\left(C_{\alpha}\right)$ if for every convergent sequence $\left\{w_{n}\right\}$ in $\mathcal{W}$ with $w_{n} \rightarrow w$ and $\alpha\left(w_{n}, w_{n+1}\right) \geq 1$ for all $n \in \mathbb{N}$, there is a subsequence $\left\{w_{n_{j}}\right\}$ of $\left\{w_{n}\right\}$ such that $\alpha\left(w_{n_{j}}, w\right) \geq 1$ for all $j \in \mathbb{N}$. Also, $\mathcal{S}$ is called $\alpha$-admissible if for all $w \in \mathcal{W}$ and $w^{\prime} \in \mathcal{S}(w)$ with $\alpha\left(w, w^{\prime}\right) \geq 1$, we have $\alpha\left(w^{\prime}, w^{\prime \prime}\right) \geq 1$ for all $w^{\prime \prime} \in \mathcal{S}\left(w^{\prime}\right)$ [45]. Finally, $w \in \mathcal{W}$ is called the endpoint of $\mathcal{S}: \mathcal{W} \rightarrow \mathcal{P}(\mathcal{W})$ if $\mathcal{S}(w)=\{w\}$ [46]. Besides, we say that $\mathcal{S}$ has an approximate endpoint property if $\inf _{w \in \mathcal{W}} \sup _{z \in \mathcal{S} w} d_{\mathcal{W}}(w, z)=0$ [46]. We need the following results.

Theorem 1 ([47]) Let $\mathcal{W}$ be a separable Banach space, let $\mathcal{S}:[0,1] \times \mathcal{W} \rightarrow \mathcal{P}_{\text {cmp,cvx }}(\mathcal{W})$ be an $\mathcal{L}^{1}$-Carathéodory set-valued map, and let $\Xi: \mathcal{L}_{\mathcal{W}}^{1}([0,1]) \rightarrow \mathcal{C}_{\mathcal{W}}([0,1])$ be a linear continuous map. Then the composition $\Xi \circ(\mathcal{S E L})_{\mathcal{S}}: \mathcal{C}_{\mathcal{W}}([0,1]) \rightarrow \mathcal{P}_{\text {cmp,cvx }}\left(\mathcal{C}_{\mathcal{W}}([0,1])\right)$ is a new operator in $\mathcal{C}_{\mathcal{W}}([0,1]) \times \mathcal{C}_{\mathcal{W}}([0,1])$ with action $w \mapsto\left(\Xi \circ(\mathcal{S E L})_{\mathcal{S}}\right)(w)=\Xi\left((\mathcal{S E})_{\mathcal{S}, w}\right)$ having the closed-graph property.

Theorem 2 ([48]) Let $\mathcal{W}$ be a Banach algebra. Assume that the following statements for a single-valued map $\Phi_{1}^{*}: \mathcal{W} \rightarrow \mathcal{W}$ and a set-valued map $\Phi_{2}^{*}: \mathcal{W} \rightarrow \mathcal{P}_{\text {cmp,cvx }}(\mathcal{W})$ are valid:

(i) $\Phi_{1}^{*}$ is Lipschitzian with constant $l^{*}$;

(ii) $\Phi_{2}^{*}$ is upper semicontinuous and compact;

(iii) $2 l^{*} \hat{\Delta}<1$, where $\hat{\Delta}=\left\|\Phi_{2}^{*}(\mathcal{W})\right\|$.

Then either (a) the set $\mathcal{O}^{*}=\left\{v^{*} \in \mathcal{W} \mid \alpha_{0} v^{*} \in\left(\Phi_{1}^{*} v^{*}\right)\left(\Phi_{2}^{*} v^{*}\right), \alpha_{0}>1\right\}$ is not bounded, or (b) there is an element belonging to $\mathcal{W}$ satisfying the inclusion $w \in\left(\Phi_{1}^{*} w\right)\left(\Phi_{2}^{*} w\right)$.

Theorem 3 ([45]) Let $\left(\mathcal{W}, d_{\mathcal{W}}\right)$ be a complete metric space. Assume that $\alpha$ is a nonnegative map on $\mathcal{W} \times \mathcal{W}, \psi \in \Psi$ is a strictly increasing map, and $\mathcal{S}: \mathcal{W} \rightarrow \mathcal{P}_{\text {cls,bnd }}(\mathcal{W})$ is an $\alpha$ admissible and $\alpha-\psi$-contractive set-valued map such that $\alpha\left(w, w^{\prime}\right) \geq 1$ for some $w \in \mathcal{W}$ and $w^{\prime} \in \mathcal{S}(w)$. Then $\mathcal{S}$ has a fixed point whenever the space $\mathcal{W}$ has the property $\left(C_{\alpha}\right)$.

Theorem $4([46])$ Let $\left(\mathcal{W}, d_{\mathcal{W}}\right)$ be a complete metric space, and let $\psi:[0, \infty) \rightarrow[0, \infty)$ be upper semicontinuous such that $\psi(s)<s$ and $\liminf _{s \rightarrow \infty}(s-\psi(s))>0$ for each $s>0$. Also, suppose that $\mathcal{S}: \mathcal{W} \rightarrow \mathcal{P}_{\text {cls,bnd }}(\mathcal{W})$ is such that $\operatorname{PH}_{d_{\mathcal{W}}}\left(\mathcal{S}_{w}, \mathcal{S} w^{\prime}\right) \leq \psi\left(d_{\mathcal{W}}\left(w, w^{\prime}\right)\right)$ for all $w, w^{\prime} \in \mathcal{W}$. Then $\mathcal{S}$ has a unique endpoint if and only if $\mathcal{S}$ has the approximate endpoint property. 


\section{Main results}

Now we are ready to state and prove our main results. Consider the Banach space $\mathcal{W}=$ $\left\{w(s): w(s) \in \mathcal{C}_{\mathbb{R}}([0,1])\right\}$ with norm $\|w\|_{\mathcal{W}}=\sup _{s \in[0,1]}|w(s)|$. Note that $(\mathcal{W},\|\cdot\| \mathcal{W})$ with multiplication given by $\left(w \cdot w^{\prime}\right)(s)=w(s) w^{\prime}(s)$ is a Banach algebra. Now consider the nonzero constants

$$
\begin{aligned}
& \tilde{\Omega}_{1}:=1-e^{-p_{2}}+\frac{p^{\xi}}{\Gamma(\xi+1)}-\int_{0}^{p} \frac{(p-r)^{\xi-1}}{\Gamma(\xi)} e^{-p_{2} r} \mathrm{~d} r \neq 0, \\
& \tilde{\Omega}_{2}:=p_{2}-1+e^{-p_{2}}+\frac{p_{2} p^{\xi+1}}{\Gamma(\xi+2)}-\frac{p^{\xi}}{\Gamma(\xi+1)}+\int_{0}^{p} \frac{(p-r)^{\xi-1}}{\Gamma(\xi)} e^{-p_{2} r} \mathrm{~d} r \neq 0, \\
& \Delta^{*}:=\tilde{\Omega}_{1}+\tilde{\Omega}_{2}=p_{2}\left(1+\frac{p^{\xi+1}}{\Gamma(\xi+2)}\right) \neq 0 .
\end{aligned}
$$

Lemma 5 Let $\tilde{a} \in \mathcal{W}$. Then $w_{0}$ is a solution function for the fractional sequential hybrid differential equation

$$
p_{1}\left({ }^{c} D_{0^{+}}^{\varrho}+p_{2}{ }^{c} D_{0^{+}}^{\varrho-1}\right)\left[\frac{w(s)}{\zeta\left(s, w(s),{ }^{R} I_{0^{+}}^{\gamma} w(s)\right)}\right]=\tilde{a}(s), \quad s \in[0,1], \varrho \in(2,3]
$$

with three-point hybrid integro-derivative boundary conditions

$$
\left\{\begin{array}{l}
{\left.\left[\frac{w(s)}{\zeta\left(s, w(s),{ }^{R} I_{0^{+}}^{\gamma} w(s)\right)}\right]\right|_{s=0}=0,} \\
\left.{ }^{c} D_{0^{+}}^{1}\left[\frac{w(s)}{\zeta\left(s, w(s),{ }^{R} I_{0^{+}}^{\gamma} w(s)\right.}\right]\right|_{s=0}+\left.{ }^{c} D_{0^{+}}^{2}\left[\frac{w(s)}{\zeta\left(s, w(s),{ }^{R} I_{0^{+}}^{\gamma} w(s)\right.}\right]\right|_{s=0}=0, \\
{\left.\left[\frac{w(s)}{\zeta\left(s, w(s),{ }^{\prime} I_{0^{+}}^{\gamma} w(s)\right)}\right]\right|_{s=1}+\left.{ }^{R} I_{0^{+}}^{\xi}\left[\frac{w(s)}{\zeta\left(s, w(s), R_{0^{+}}^{\gamma} w(s)\right)}\right]\right|_{s=p}=0,}
\end{array}\right.
$$

if and only if $w_{0}$ is a solution of the integral equation

$$
\begin{aligned}
w(s)= & \zeta\left(s, w(s),{ }^{R} I_{0^{+}}^{\gamma} w(s)\right)\left(\frac{1}{p_{1}} \int_{0}^{s} e^{-p_{2}(s-r)} \int_{0}^{r} \frac{(r-m)^{\varrho-2}}{\Gamma(\varrho-1)} \tilde{a}(m) \mathrm{d} m \mathrm{~d} r\right. \\
& +\frac{1-e^{-p_{2} s}+\left(p_{2}^{2}-p_{2}\right) s}{p_{1}\left(\tilde{\Omega}_{2}-p_{2} \Delta^{*}\right)}\left[\int_{0}^{1} e^{-p_{2}(1-r)} \int_{0}^{r} \frac{(r-m)^{\varrho-2}}{\Gamma(\varrho-1)} \tilde{a}(m) \mathrm{d} m \mathrm{~d} r\right. \\
& \left.\left.+\int_{0}^{p} \frac{(p-r)^{\xi-1}}{\Gamma(\xi)} \int_{0}^{r} e^{-p_{2}(r-\tau)} \int_{0}^{\tau} \frac{(\tau-m)^{\varrho-2}}{\Gamma(\varrho-1)} \tilde{a}(m) \mathrm{d} m \mathrm{~d} \tau \mathrm{d} r\right]\right),
\end{aligned}
$$

where $\tilde{\Omega}_{1}, \tilde{\Omega}_{2}$, and $\Delta^{*}$ are given in (5).

Proof First, suppose that $w_{0}$ is a solution for the sequential hybrid equation (6). Then

$$
p_{1}{ }^{c} D_{0^{+}}^{\varrho}\left(1+p_{2}{ }^{c} D_{0^{+}}^{-1}\right)\left[\frac{w_{0}(s)}{\zeta\left(s, w_{0}(s),{ }^{R} I_{0^{+}}^{\gamma} w_{0}(s)\right)}\right]=\tilde{a}(s) .
$$

By taking the $\varrho$ th-order Riemann-Liouville integral on this equality, we obtain

$$
p_{1}\left(1+p_{2}{ }^{c} D_{0^{+}}^{-1}\right)\left[\frac{w_{0}(s)}{\zeta\left(s, w_{0}(s),{ }^{R} I_{0^{+}}^{\gamma} w_{0}(s)\right)}\right]={ }^{R} I_{0^{+}}^{\varrho} \tilde{a}(s)+\tilde{m}_{0}+\tilde{m}_{1} s+\tilde{m}_{2} s^{2} .
$$


Hence

$$
\begin{aligned}
p_{1}\left[\frac{w_{0}(s)}{\zeta\left(s, w_{0}(s),{ }^{R} I_{0^{+}}^{\gamma} w_{0}(s)\right)}\right]= & -p_{1} p_{2} \int_{0}^{s}\left[\frac{w_{0}(r)}{\zeta\left(r, w_{0}(r),{ }^{R} I_{0^{+}}^{\gamma} w_{0}(r)\right)}\right] \mathrm{d} r \\
& +{ }^{R} I_{0^{+}}^{\varrho} \tilde{a}(s)+\tilde{m}_{0}+\tilde{m}_{1} s+\tilde{m}_{2} s^{2} .
\end{aligned}
$$

The last equality yields $p_{1}\left[\frac{w_{0}(0)}{\zeta\left(s, w_{0}(0),{ }^{R}{ }_{0^{+}}^{\gamma} w_{0}(0)\right)}\right]=\tilde{m}_{0}$. On the other hand, taking the first-order Caputo derivative of Equation (9) with respect to $s$, we get

$$
\begin{aligned}
p_{1}{ }^{c} D_{0^{+}}^{1}\left[\frac{w_{0}(s)}{\zeta\left(s, w_{0}(s),{ }^{R} I_{0^{+}}^{\gamma} w_{0}(s)\right)}\right] \\
\quad=-p_{1} p_{2}\left[\frac{w_{0}(s)}{\zeta\left(s, w_{0}(s),{ }^{R} I_{0^{+}}^{\gamma} w_{0}(s)\right)}\right]+{ }^{R} I_{0^{+}}^{\varrho-1} \tilde{a}(s)+\tilde{m}_{1}+2 \tilde{m}_{2} s .
\end{aligned}
$$

Now we multiply both sides of the last equality by $e^{p_{2} s}$ :

$$
\begin{aligned}
& p_{1}{ }^{c} D_{0^{+}}^{1}\left[\frac{w_{0}(s)}{\zeta\left(s, w_{0}(s),{ }^{R} I_{0^{+}}^{\gamma} w_{0}(s)\right)}\right] e^{p_{2} s}+p_{1} p_{2}\left[\frac{w_{0}(s)}{\zeta\left(s, w_{0}(s),{ }^{R} I_{0^{+}}^{\gamma} w_{0}(s)\right)}\right] e^{p_{2} s} \\
& =e^{p_{2} s R} I_{0^{+}}^{Q-1} \tilde{a}(s)+\tilde{m}_{1} e^{p_{2} s}+2 \tilde{m}_{2} s e^{p_{2} s}
\end{aligned}
$$

By direct computations, after some simplifications, we get

$$
\begin{aligned}
\frac{w_{0}(s)}{\zeta\left(s, w_{0}(s),{ }^{R} I_{0^{+}}^{\gamma} w_{0}(s)\right)}= & \frac{1}{p_{1}} \int_{0}^{s} e^{-p_{2}(s-r)} \int_{0}^{r} \frac{(r-m)^{\varrho-2}}{\Gamma(\varrho-1)} \tilde{a}(m) \mathrm{d} m \mathrm{~d} r \\
& +\frac{\tilde{m}_{0}}{p_{1}} e^{-p_{2} s}+\frac{\tilde{m}_{1}}{p_{1} p_{2}}\left(1-e^{-p_{2} s}\right)+\frac{2 \tilde{m}_{2}}{p_{1} p_{2}^{2}}\left(p_{2} s-1+e^{-p_{2} s}\right) .
\end{aligned}
$$

Finally, in view of three-point hybrid boundary conditions, we obtain $\tilde{m}_{0}=0$,

$$
\begin{aligned}
\tilde{m}_{1}= & \frac{p_{2}^{2}}{\tilde{\Omega}_{2}-p_{2} \Delta^{*}}\left(\int_{0}^{1} e^{-p_{2}(1-r)} \int_{0}^{r} \frac{(r-m)^{\varrho-2}}{\Gamma(\varrho-1)} \tilde{a}(m) \mathrm{d} m \mathrm{~d} r\right. \\
& \left.+\int_{0}^{p} \frac{(p-r)^{\xi-1}}{\Gamma(\xi)} \int_{0}^{r} e^{-p_{2}(r-\tau)} \int_{0}^{\tau} \frac{(\tau-m)^{\varrho-2}}{\Gamma(\varrho-1)} \tilde{a}(m) \mathrm{d} m \mathrm{~d} \tau \mathrm{d} r\right),
\end{aligned}
$$

and

$$
\begin{aligned}
\tilde{m}_{2}= & -\frac{p_{2}^{2}\left(1-p_{2}\right)}{2\left(\tilde{\Omega}_{2}-p_{2} \Delta^{*}\right)}\left(\int_{0}^{1} e^{-p_{2}(1-r)} \int_{0}^{r} \frac{(r-m)^{\varrho-2}}{\Gamma(\varrho-1)} \tilde{a}(m) \mathrm{d} m \mathrm{~d} r\right. \\
& \left.+\int_{0}^{p} \frac{(p-r)^{\xi-1}}{\Gamma(\xi)} \int_{0}^{r} e^{-p_{2}(r-\tau)} \int_{0}^{\tau} \frac{(\tau-m)^{\varrho-2}}{\Gamma(\varrho-1)} \tilde{a}(m) \mathrm{d} m \mathrm{~d} \tau \mathrm{d} r\right) .
\end{aligned}
$$


If we insert the values $\tilde{m}_{0}, \tilde{m}_{1}$, and $\tilde{m}_{2}$ into Equation (10), then

$$
\begin{aligned}
w_{0}(s)= & \zeta\left(s, w_{0}(s),{ }^{R} I_{0^{+}}^{\gamma} w_{0}(s)\right)\left(\frac{1}{p_{1}} \int_{0}^{s} e^{-p_{2}(s-r)} \int_{0}^{r} \frac{(r-m)^{\varrho-2}}{\Gamma(\varrho-1)} \tilde{a}(m) \mathrm{d} m \mathrm{~d} r\right. \\
& +\frac{1-e^{-p_{2} s}+\left(p_{2}^{2}-p_{2}\right) s}{p_{1}\left(\tilde{\Omega}_{2}-p_{2} \Delta^{*}\right)}\left[\int_{0}^{1} e^{-p_{2}(1-r)} \int_{0}^{r} \frac{(r-m)^{\varrho-2}}{\Gamma(\varrho-1)} \tilde{a}(m) \mathrm{d} m \mathrm{~d} r\right. \\
& \left.\left.+\int_{0}^{p} \frac{(p-r)^{\xi-1}}{\Gamma(\xi)} \int_{0}^{r} e^{-p_{2}(r-\tau)} \int_{0}^{\tau} \frac{(\tau-m)^{\varrho-2}}{\Gamma(\varrho-1)} \tilde{a}(m) \mathrm{d} m \mathrm{~d} \tau \mathrm{d} r\right]\right) .
\end{aligned}
$$

This shows that the function $w_{0}$ satisfies the integral equation (8). For the next part, it is easy to check that $w_{0}$ is a solution for the sequential BVP (6)-(7) whenever $w_{0}$ satisfies the integral equation (8).

Now, in the following theorem, we deal with some useful estimates.

Theorem 6 Let $\tilde{a}:[0,1] \rightarrow \mathbb{R}$ be a continuous function such that $\|\tilde{a}\|=\sup _{s \in[0,1]}|\tilde{a}(s)|$. Then we have:

$$
\begin{aligned}
& \text { (A1) }\left|\int_{0}^{p} \frac{(p-r)^{\xi-1}}{\Gamma(\xi)} \int_{0}^{r} e^{-p_{2}(r-\tau)} \int_{0}^{\tau} \frac{(\tau-m)^{\varrho-2}}{\Gamma(\varrho-1)} \tilde{a}(m) \mathrm{d} m \mathrm{~d} \tau \mathrm{d} r\right| \leq \frac{p^{\varrho+\xi-1}\left(p_{2} p+e^{-p_{2} p}-1\right)}{p_{2}^{2} \Gamma(\varrho) \Gamma(\xi)}\|\tilde{a}\|, \\
& \text { (A2) }\left|\int_{0}^{s} e^{-p_{2}(s-r)} \int_{0}^{r} \frac{(r-m)^{\varrho-2}}{\Gamma(\varrho-1)} \tilde{a}(m) \mathrm{d} m \mathrm{~d} r\right| \leq \frac{1}{p_{2} \Gamma(\varrho)}\left(1-e^{-p_{2}}\right)\|\tilde{a}\|, \\
& \text { (A3) }\left|\int_{0}^{1} e^{-p_{2}(1-r)} \int_{0}^{r} \frac{(r-m)^{\varrho-2}}{\Gamma(\varrho-1)} \tilde{a}(m) \mathrm{d} m \mathrm{~d} r\right| \leq \frac{1}{p_{2} \Gamma(\varrho)}\left(1-e^{-p_{2}}\right)\|\tilde{a}\| .
\end{aligned}
$$

Proof (A1) First of all, an easy computation yields

$$
\int_{0}^{\tau} \frac{(\tau-m)^{\varrho-2}}{\Gamma(\varrho-1)} \mathrm{d} m=-\left.\frac{(\tau-m)^{\varrho-1}}{\Gamma(\varrho)}\right|_{0} ^{\tau}=\frac{\tau^{\varrho-1}}{\Gamma(\varrho)}
$$

We further have

$$
\begin{aligned}
\int_{0}^{r} e^{-p_{2}(r-\tau)} \int_{0}^{\tau} \frac{(\tau-m)^{\varrho-2}}{\Gamma(\varrho-1)} \mathrm{d} m \mathrm{~d} \tau & =\int_{0}^{r} e^{-p_{2}(r-\tau)} \frac{\tau^{\varrho-1}}{\Gamma(\varrho)} \mathrm{d} \tau \\
& \leq \frac{r^{\varrho-1}}{\Gamma(\varrho)} \int_{0}^{r} e^{-p_{2}(r-\tau)} \mathrm{d} \tau \\
& =\left.\frac{r^{\varrho-1}}{\Gamma(\varrho)}\left(-\frac{1}{p_{2}} e^{-p_{2}(r-\tau)}\right)\right|_{0} ^{r} \\
& =-\frac{r^{\varrho-1}}{p_{2} \Gamma(\varrho)}\left(1-e^{-p_{2} r}\right) .
\end{aligned}
$$

Now combining the obtained results, we get

$$
\begin{aligned}
& \left|\int_{0}^{p} \frac{(p-r)^{\xi-1}}{\Gamma(\xi)} \int_{0}^{r} e^{-p_{2}(r-\tau)} \int_{0}^{\tau} \frac{(\tau-m)^{\varrho-2}}{\Gamma(\varrho-1)} \tilde{a}(m) \mathrm{d} m \mathrm{~d} \tau \mathrm{d} r\right| \\
& \quad \leq\|\tilde{a}\| \int_{0}^{p} \frac{(p-r)^{\xi-1}}{\Gamma(\xi)} \frac{r^{\varrho-1}\left(1-e^{-p_{2} r}\right)}{p_{2} \Gamma(\varrho)} \mathrm{d} r \\
& \quad \leq\|\tilde{a}\| \frac{1}{p_{2} \Gamma(\xi) \Gamma(\varrho)} \int_{0}^{p} p^{\xi-1} p^{\varrho-1}\left(1-e^{-p_{2} r}\right) \mathrm{d} r \\
& \quad \leq\|\tilde{a}\| \frac{p^{\varrho+\xi-1}}{p_{2} \Gamma(\xi) \Gamma(\varrho)} \int_{0}^{p}\left(1-e^{-p_{2} r}\right) \mathrm{d} r
\end{aligned}
$$




$$
\begin{aligned}
& =\|\tilde{a}\| \frac{p^{\varrho+\xi-1}}{p_{2} \Gamma(\xi) \Gamma(\varrho)}\left(p+\frac{1}{p_{2}} e^{-p_{2} p}-\frac{1}{p_{2}}\right) \\
& =\frac{p^{\varrho+\xi-1}}{p_{2}^{2} \Gamma(\xi) \Gamma(\varrho)}\left(p_{2} p+e^{-p_{2} p}-1\right)\|\tilde{a}\| .
\end{aligned}
$$

This completes the proof of (A1).

(A2) Similarly to Equation (12), we have $\int_{0}^{r} \frac{(r-m)^{\varrho-2}}{\Gamma(\varrho-1)} \mathrm{d} m=\frac{r^{\varrho-1}}{\Gamma(\varrho)}$. Hence

$$
\begin{aligned}
\int_{0}^{s} e^{-p_{2}(s-r)} \int_{0}^{r} \frac{(r-m)^{\varrho-2}}{\Gamma(\varrho-1)} \mathrm{d} m \mathrm{~d} r & =\int_{0}^{s} e^{-p_{2}(s-r)} \frac{r^{\varrho-1}}{\Gamma(\varrho)} \mathrm{d} r \\
& \leq \frac{s^{\varrho-1}}{\Gamma(\varrho)} \int_{0}^{s} e^{-p_{2}(s-r)} \mathrm{d} r \\
& \leq\left.\frac{1}{\Gamma(\varrho)}\left(-\frac{1}{p_{2}} e^{-p_{2}(s-r)}\right)\right|_{0} ^{s}=-\frac{1}{p_{2} \Gamma(\varrho)}\left(1-e^{-p_{2} s}\right) .
\end{aligned}
$$

Thus $\left|\int_{0}^{s} e^{-p_{2}(s-r)} \int_{0}^{r} \frac{(r-m)^{\varrho^{-2}}}{\Gamma(\varrho-1)} \tilde{a}(m) \mathrm{d} m \mathrm{~d} r\right| \leq \frac{1}{p_{2} \Gamma(\varrho)}\left(1-e^{-p_{2}}\right)\|\tilde{a}\|$.

(A3) The proof of this estimate is similar to that of (A2) and so is omitted.

Definition 7 A function $w \in \mathcal{A C}_{\mathbb{R}}([0,1])$ is said to be a solution for the sequential hybrid inclusion BVP (1)-(2) if there is an integrable function $\hat{\vartheta} \in \mathcal{L}_{\mathbb{R}}^{1}([0,1])$ along with $\hat{\vartheta}(s) \in \mathcal{S}(s, w(s))$ for almost all $s \in[0,1]$ satisfying the three-point hybrid integro-derivative boundary conditions

$$
\left\{\begin{array}{l}
{\left.\left[\frac{w(s)}{\zeta\left(s, w(s),{ }^{R} I_{0^{+}}^{\gamma} w(s)\right)}\right]\right|_{s=0}=0,} \\
\left.{ }^{c} D_{0^{+}}^{1}\left[\frac{w(s)}{\zeta\left(s, w(s),{ }^{R} I_{0^{+}}^{\gamma} w(s)\right.}\right]\right|_{s=0}+\left.{ }^{c} D_{0^{+}}^{2}\left[\frac{w(s)}{\zeta\left(s, w(s),{ }^{R} I_{0^{+}}^{\gamma} w(s)\right.}\right]\right|_{s=0}=0, \\
{\left.\left[\frac{w(s)}{\zeta\left(s, w(s), R I_{0^{+}}^{\gamma} w(s)\right)}\right]\right|_{s=1}+\left.{ }^{R} I_{0^{+}}^{\xi}\left[\frac{w(s)}{\zeta\left(s, w(s), R_{0^{+}}^{\gamma} w(s)\right)}\right]\right|_{s=p}=0,}
\end{array}\right.
$$

and

$$
\begin{aligned}
w(s)= & \zeta\left(s, w(s),{ }^{R} I_{0^{+}}^{\gamma} w(s)\right)\left(\frac{1}{p_{1}} \int_{0}^{s} e^{-p_{2}(s-r)} \int_{0}^{r} \frac{(r-m)^{\varrho-2}}{\Gamma(\varrho-1)} \hat{\vartheta}(m) \mathrm{d} m \mathrm{~d} r\right. \\
& +\frac{1-e^{-p_{2} s}+\left(p_{2}^{2}-p_{2}\right) s}{p_{1}\left(\tilde{\Omega}_{2}-p_{2} \Delta^{*}\right)}\left[\int_{0}^{1} e^{-p_{2}(1-r)} \int_{0}^{r} \frac{(r-m)^{\varrho-2}}{\Gamma(\varrho-1)} \hat{\vartheta}(m) \mathrm{d} m \mathrm{~d} r\right. \\
& \left.\left.+\int_{0}^{p} \frac{(p-r)^{\xi-1}}{\Gamma(\xi)} \int_{0}^{r} e^{-p_{2}(r-\tau)} \int_{0}^{\tau} \frac{(\tau-m)^{\varrho-2}}{\Gamma(\varrho-1)} \hat{\vartheta}(m) \mathrm{d} m \mathrm{~d} \tau \mathrm{d} r\right]\right)
\end{aligned}
$$

for all $s \in[0,1]$.

Theorem 8 Suppose that $\zeta$ is a nonzero continuous real-valued map on $[0,1] \times \mathbb{R} \times \mathbb{R}$ and $\mathcal{S}:[0,1] \times \mathbb{R} \rightarrow \mathcal{P}_{\text {cmp,cvx }}(\mathbb{R})$ is a set-valued map. Moreover,

(Hyp1) there is a bounded real-valued map $v:[0,1] \rightarrow \mathbb{R}^{+}$such that

$$
\left|\zeta\left(s, w_{1}(s), w_{2}(s)\right)-\zeta\left(s, w_{1}^{\prime}(s), w_{2}^{\prime}(s)\right)\right| \leq v(s) \sum_{i=1}^{2}\left|w_{i}(s)-w_{i}^{\prime}(s)\right|
$$

for all $w_{1}, w_{2}, w_{1}^{\prime}, w_{2}^{\prime} \in \mathbb{R}$ and $s \in[0,1]$, 
(Hyp2) the set-valued map $\mathcal{S}:[0,1] \times \mathbb{R} \rightarrow \mathcal{P}_{\text {cmp,cvx }}(\mathbb{R})$ is $\mathcal{L}^{1}$-Carathéodory,

(Hyp3) there is a positive mapping $\theta \in \mathcal{L}_{\mathbb{R}^{+}}^{1}([0,1])$ such that

$$
\|\mathcal{S}(s, w)\|=\sup \{|\hat{\vartheta}|: \hat{\vartheta} \in \mathcal{S}(s, w(s))\} \leq \theta(s)
$$

for all $w \in \mathbb{R}$ and almost all $s \in[0,1]$, and

(Hyp4) there is $q \in \mathbb{R}^{+}$such that

$$
q>\frac{\zeta^{*} M\|\theta\|_{\mathcal{L}^{1}}}{1-v^{*}\left(1+\frac{1}{\Gamma(\gamma+1)}\right) M\|\theta\|_{\mathcal{L}^{1}}}
$$

where $\|\theta\|_{\mathcal{L}^{1}}=\int_{0}^{1}|\theta(r)| \mathrm{d} r, \zeta^{*}=\sup _{s \in[0,1]}|\zeta(s, 0,0)|, \nu^{*}=\sup _{s \in[0,1]}|\nu(s)|$, and

$$
\begin{aligned}
M= & \frac{\left(1-e^{-p_{2}}\right)}{p_{1} p_{2} \Gamma(\varrho)}+\frac{\left|1-e^{-p_{2}}\right|+\left|p_{2}^{2}-p_{2}\right|}{p_{1}\left|\tilde{\Omega}_{2}-p_{2} \Delta^{*}\right|} \\
& \times\left(\frac{\left(1-e^{-p_{2}}\right)}{p_{2} \Gamma(\varrho)}+\frac{p^{\varrho+\xi-1}\left(p_{2} p+e^{-p_{2} p}-1\right)}{p_{2}^{2} \Gamma(\varrho) \Gamma(\xi)}\right) .
\end{aligned}
$$

If $\nu^{*}\left(1+\frac{1}{\Gamma(\gamma+1)}\right) M\|\theta\|_{\mathcal{L}^{1}}<\frac{1}{2}$, then the sequential hybrid inclusion BVP (1)-(2) has a solution.

Proof Let $w \in \mathcal{W}$. Consider the following family of selections of the operator $\mathcal{S}$ :

$$
(\mathcal{S E L})_{\mathcal{S}, w}=\left\{\hat{\vartheta} \in \mathcal{L}^{1}([0,1]): \hat{\vartheta}(s) \in \mathcal{S}(s, w(s)) \text { for almost all } s \in[0,1]\right\}
$$

Define $\mathcal{G}: \mathcal{W} \rightarrow \mathcal{P}(\mathcal{W})$ by $\mathcal{G}(w)=\{g \in \mathcal{W}: g(s)=\rho(s)$ for $s \in[0,1]\}$, where

$$
\begin{aligned}
\rho(s)= & \zeta\left(s, w(s),{ }^{R} I_{0^{+}}^{\gamma} w(s)\right)\left(\frac{1}{p_{1}} \int_{0}^{s} e^{-p_{2}(s-r)} \int_{0}^{r} \frac{(r-m)^{\varrho-2}}{\Gamma(\varrho-1)} \hat{\vartheta}(m) \mathrm{d} m \mathrm{~d} r\right. \\
& +\frac{1-e^{-p_{2} s}+\left(p_{2}^{2}-p_{2}\right) s}{p_{1}\left(\tilde{\Omega}_{2}-p_{2} \Delta^{*}\right)}\left[\int_{0}^{1} e^{-p_{2}(1-r)} \int_{0}^{r} \frac{(r-m)^{\varrho-2}}{\Gamma(\varrho-1)} \hat{\vartheta}(m) \mathrm{d} m \mathrm{~d} r\right. \\
& \left.\left.+\int_{0}^{p} \frac{(p-r)^{\xi-1}}{\Gamma(\xi)} \int_{0}^{r} e^{-p_{2}(r-\tau)} \int_{0}^{\tau} \frac{(\tau-m)^{\varrho-2}}{\Gamma(\varrho-1)} \hat{\vartheta}(m) \mathrm{d} m \mathrm{~d} \tau \mathrm{d} r\right]\right)
\end{aligned}
$$

for some $\hat{\vartheta} \in(\mathcal{S E L})_{\mathcal{S}, w}$. It is known that an element $g_{0}$ of $\mathcal{W}$ is a solution of the sequential hybrid inclusion BVP (1)-(2) if and only if $g_{0}$ is a fixed point of $\mathcal{G}$. As we said in the light of Lemma 5, we introduce two mappings $\Phi_{1}^{*}: \mathcal{W} \rightarrow \mathcal{W}$ and $\Phi_{2}^{*}: \mathcal{W} \rightarrow \mathcal{P}(\mathcal{W})$ defined by $\left(\Phi_{1}^{*} w\right)(s)=\zeta\left(s, w(s),{ }^{R} I_{0^{+}}^{\gamma} w(s)\right)$ and $\left(\Phi_{2}^{*} w\right)(s)=\{v \in \mathcal{W}: v(s)=b(s)$ for $s \in[0,1]\}$, where

$$
\begin{aligned}
b(s)= & \frac{1}{p_{1}} \int_{0}^{s} e^{-p_{2}(s-r)} \int_{0}^{r} \frac{(r-m)^{\varrho-2}}{\Gamma(\varrho-1)} \hat{\vartheta}(m) \mathrm{d} m \mathrm{~d} r \\
& +\frac{1-e^{-p_{2} s}+\left(p_{2}^{2}-p_{2}\right) s}{p_{1}\left(\tilde{\Omega}_{2}-p_{2} \Delta^{*}\right)}\left[\int_{0}^{1} e^{-p_{2}(1-r)} \int_{0}^{r} \frac{(r-m)^{\varrho-2}}{\Gamma(\varrho-1)} \hat{\vartheta}(m) \mathrm{d} m \mathrm{~d} r\right. \\
& \left.+\int_{0}^{p} \frac{(p-r)^{\xi-1}}{\Gamma(\xi)} \int_{0}^{r} e^{-p_{2}(r-\tau)} \int_{0}^{\tau} \frac{(\tau-m)^{\varrho-2}}{\Gamma(\varrho-1)} \hat{\vartheta}(m) \mathrm{d} m \mathrm{~d} \tau \mathrm{d} r\right]
\end{aligned}
$$


for some $\hat{\vartheta} \in(\mathcal{S E} \mathcal{L})_{\mathcal{S}, w}$. Then we can express the product equation $\mathcal{G}(w)=\Phi_{1}^{*} w \Phi_{2}^{*} w$. The main objective herein is to show that $\Phi_{1}^{*}$ and $\Phi_{2}^{*}$ satisfy all assumptions of Theorem 2 . We first proceed by proving that the operator $\Phi_{1}^{*}$ is Lipschitzian on $\mathcal{W}$. Let $w_{1}, w_{2} \in \mathcal{W}$ be arbitrary elements. Under hypothesis (Hyp1), it follows that

$$
\begin{aligned}
\left|\left(\Phi_{1}^{*} w_{1}\right)(s)-\left(\Phi_{1}^{*} w_{2}\right)(s)\right| & =\left|\zeta\left(s, w_{1}(s),{ }^{R} I_{0^{+}}^{\gamma} w_{1}(s)\right)-\zeta\left(s, w_{2}(s),{ }^{R} I_{0^{+}}^{\gamma} w_{2}(s)\right)\right| \\
& \leq v(s)\left(\left|w_{1}(s)-w_{2}(s)\right|+\frac{1}{\Gamma(\gamma+1)}\left|w_{1}(s)-w_{2}(s)\right|\right) \\
& =v(s)\left(1+\frac{1}{\Gamma(\gamma+1)}\right)\left|w_{1}(s)-w_{2}(s)\right|
\end{aligned}
$$

for all $s \in[0,1]$. Therefore we hqve $\left\|\Phi_{1}^{*} w_{1}-\Phi_{1}^{*} w_{2}\right\|_{\mathcal{W}} \leq v^{*}\left(1+\frac{1}{\Gamma(\gamma+1)}\right)\left\|w_{1}-w_{2}\right\|_{\mathcal{W}}$ for all $w_{1}, w_{2} \in \mathcal{W}$, that is, the single-valued operator $\Phi_{1}^{*}$ is Lipschitzian with constant $v^{*}(1+$ $\left.\frac{1}{\Gamma(\gamma+1)}\right)$. In the subsequent step, we will check that the set-valued map $\Phi_{2}^{*}$ is convex-valued. Let $w_{1}, w_{2} \in \Phi_{2}^{*} w$. We choose $\hat{\vartheta_{1}}, \hat{\vartheta_{2}} \in(\mathcal{S E} \mathcal{L})_{\mathcal{S}, w}$ such that

$$
\begin{aligned}
w_{i}(s)= & \frac{1}{p_{1}} \int_{0}^{s} e^{-p_{2}(s-r)} \int_{0}^{r} \frac{(r-m)^{\varrho-2}}{\Gamma(\varrho-1)} \hat{\vartheta}_{i}(m) \mathrm{d} m \mathrm{~d} r \\
& +\frac{1-e^{-p_{2} s}+\left(p_{2}^{2}-p_{2}\right) s}{p_{1}\left(\tilde{\Omega}_{2}-p_{2} \Delta^{*}\right)}\left[\int_{0}^{1} e^{-p_{2}(1-r)} \int_{0}^{r} \frac{(r-m)^{\varrho-2}}{\Gamma(\varrho-1)} \hat{\vartheta}_{i}(m) \mathrm{d} m \mathrm{~d} r\right. \\
& \left.+\int_{0}^{p} \frac{(p-r)^{\xi-1}}{\Gamma(\xi)} \int_{0}^{r} e^{-p_{2}(r-\tau)} \int_{0}^{\tau} \frac{(\tau-m)^{\varrho-2}}{\Gamma(\varrho-1)} \hat{\vartheta}_{i}(m) \mathrm{d} m \mathrm{~d} \tau \mathrm{d} r\right]
\end{aligned}
$$

for almost all $s \in[0,1]$ and $i=1,2$. Let $\lambda \in(0,1)$. Then we can write

$$
\begin{aligned}
\lambda w_{1}(s) & +(1-\lambda) w_{2}(s) \\
= & \frac{1}{p_{1}} \int_{0}^{s} e^{-p_{2}(s-r)} \int_{0}^{r} \frac{(r-m)^{\varrho-2}}{\Gamma(\varrho-1)}\left[\lambda \hat{\vartheta}_{1}(m)+(1-\lambda) \hat{\vartheta}_{2}(m)\right] \mathrm{d} m \mathrm{~d} r \\
& +\frac{1-e^{-p_{2} s}+\left(p_{2}^{2}-p_{2}\right) s}{p_{1}\left(\tilde{\Omega}_{2}-p_{2} \Delta^{*}\right)} \\
& \times\left[\int_{0}^{1} e^{-p_{2}(1-r)} \int_{0}^{r} \frac{(r-m)^{\varrho-2}}{\Gamma(\varrho-1)}\left[\lambda \hat{\vartheta}_{1}(m)+(1-\lambda) \hat{\vartheta}_{2}(m)\right] \mathrm{d} m \mathrm{~d} r\right. \\
& \left.+\int_{0}^{p} \frac{(p-r)^{\xi-1}}{\Gamma(\xi)} \int_{0}^{r} e^{-p_{2}(r-\tau)} \int_{0}^{\tau} \frac{(\tau-m)^{\varrho-2}}{\Gamma(\varrho-1)}\left[\lambda \hat{\vartheta}_{1}(m)+(1-\lambda) \hat{\vartheta}_{2}(m)\right] \mathrm{d} m \mathrm{~d} \tau \mathrm{d} r\right]
\end{aligned}
$$

for almost all $s \in[0,1]$. Since $\mathcal{S}$ has convex values, $(\mathcal{S E} \mathcal{L})_{\mathcal{S}, w}$ is a convex set. By this point we find that $\lambda \hat{\vartheta}_{1}(s)+(1-\lambda) \hat{\vartheta}_{2}(s) \in(\mathcal{S E L})_{\mathcal{S}, w}$ for any $s \in[0,1]$, and so $\Phi_{2}^{*} w$ belongs to $\mathcal{P}_{\text {cvx }}(\mathcal{W})$ for all $w \in \mathcal{W}$.

Now we prove the complete continuity of $\Phi_{2}^{*}$ on $\mathcal{W}$. We need to prove the equicontinuity and uniform boundedness of the set $\Phi_{2}^{*}(\mathcal{W})$. To this aim, we first show that $\Phi_{2}^{*}$ maps each bounded set to a bounded subset of $\mathcal{W}$. For $\tilde{\varepsilon} \in \mathbb{R}^{+}$, consider the bounded ball $\mathcal{V}_{\tilde{\varepsilon}}=\left\{w \in \mathcal{W}:\|w\|_{\mathcal{W}} \leq \tilde{\varepsilon}\right\}$. For $w \in \mathcal{V}_{\tilde{\varepsilon}}$ and $v \in \Phi_{2}^{*} w$, choose $\hat{\vartheta} \in(\mathcal{S E} \mathcal{L})_{\mathcal{S}, w}$ such 
that

$$
\begin{aligned}
v(s)= & \frac{1}{p_{1}} \int_{0}^{s} e^{-p_{2}(s-r)} \int_{0}^{r} \frac{(r-m)^{\varrho-2}}{\Gamma(\varrho-1)} \hat{\vartheta}(m) \mathrm{d} m \mathrm{~d} r \\
& +\frac{1-e^{-p_{2} s}+\left(p_{2}^{2}-p_{2}\right) s}{p_{1}\left(\tilde{\Omega}_{2}-p_{2} \Delta^{*}\right)}\left[\int_{0}^{1} e^{-p_{2}(1-r)} \int_{0}^{r} \frac{(r-m)^{\varrho-2}}{\Gamma(\varrho-1)} \hat{\vartheta}(m) \mathrm{d} m \mathrm{~d} r\right. \\
& \left.+\int_{0}^{p} \frac{(p-r)^{\xi-1}}{\Gamma(\xi)} \int_{0}^{r} e^{-p_{2}(r-\tau)} \int_{0}^{\tau} \frac{(\tau-m)^{\varrho-2}}{\Gamma(\varrho-1)} \hat{\vartheta}(m) \mathrm{d} m \mathrm{~d} \tau \mathrm{d} r\right]
\end{aligned}
$$

for all $s \in[0,1]$. Then we can estimate the function $v$ by

$$
\begin{aligned}
|v(s)| \leq & \frac{1}{p_{1}} \int_{0}^{s} e^{-p_{2}(s-r)} \int_{0}^{r} \frac{(r-m)^{\varrho-2}}{\Gamma(\varrho-1)}|\hat{\vartheta}(m)| \mathrm{d} m \mathrm{~d} r \\
& +\frac{\left|1-e^{-p_{2} s}\right|+\left|p_{2}^{2}-p_{2}\right| s}{p_{1}\left|\tilde{\Omega}_{2}-p_{2} \Delta^{*}\right|}\left[\int_{0}^{1} e^{-p_{2}(1-r)} \int_{0}^{r} \frac{(r-m)^{\varrho-2}}{\Gamma(\varrho-1)}|\hat{\vartheta}(m)| \mathrm{d} m \mathrm{~d} r\right. \\
& \left.+\int_{0}^{p} \frac{(p-r)^{\xi-1}}{\Gamma(\xi)} \int_{0}^{r} e^{-p_{2}(r-\tau)} \int_{0}^{\tau} \frac{(\tau-m)^{\varrho-2}}{\Gamma(\varrho-1)}|\hat{\vartheta}(m)| \mathrm{d} m \mathrm{~d} \tau \mathrm{d} r\right] \\
\leq & \frac{1}{p_{1}} \int_{0}^{s} e^{-p_{2}(s-r)} \int_{0}^{r} \frac{(r-m)^{\varrho-2}}{\Gamma(\varrho-1)} \theta(m) \mathrm{d} m \mathrm{~d} r \\
& +\frac{\left|1-e^{-p_{2} s}\right|+\left|p_{2}^{2}-p_{2}\right| s}{p_{1}\left|\tilde{\Omega}_{2}-p_{2} \Delta^{*}\right|}\left[\int_{0}^{1} e^{-p_{2}(1-r)} \int_{0}^{r} \frac{(r-m)^{\varrho-2}}{\Gamma(\varrho-1)} \theta(m) \mathrm{d} m \mathrm{~d} r\right. \\
& \left.+\int_{0}^{p} \frac{(p-r)^{\xi-1}}{\Gamma(\xi)} \int_{0}^{r} e^{-p_{2}(r-\tau)} \int_{0}^{\tau} \frac{(\tau-m)^{\varrho-2}}{\Gamma(\varrho-1)} \theta(m) \mathrm{d} m \mathrm{~d} \tau \mathrm{d} r\right] \\
\leq & {\left[\frac{\left(1-e^{-p_{2}}\right)}{p_{1} p_{2} \Gamma(\varrho)}+\frac{\left|1-e^{-p_{2}}\right|+\left|p_{2}^{2}-p_{2}\right|}{p_{1}\left|\tilde{\Omega}_{2}-p_{2} \Delta^{*}\right|}\right.} \\
& \left.\times\left(\frac{\left(1-e^{-p_{2}}\right)}{p_{2} \Gamma(\varrho)}+\frac{p^{\varrho+\xi-1}\left(p_{2} p+e^{-p_{2} p}-1\right)}{p_{2}^{2} \Gamma(\varrho) \Gamma(\xi)}\right)\right]\|\theta\|_{\mathcal{L}^{1}}=M\|\theta\|_{\mathcal{L}^{1}},
\end{aligned}
$$

where $M$ is given in (14). Consequently, $\|v\| \leq M\|\theta\|_{\mathcal{L}^{1}}$, which means that $\Phi_{2}^{*}(\mathcal{W})$ is uniformly bounded. Now we claim that the operator $\Phi_{2}^{*}$ maps each bounded set to an equicontinuous subset. Let $w \in \mathcal{V}_{\tilde{\varepsilon}}$ and $v \in \Phi_{2}^{*} w$. Select $\hat{\vartheta} \in(\mathcal{S E} \mathcal{L})_{\mathcal{S}, w}$ such that

$$
\begin{aligned}
v(s)= & \frac{1}{p_{1}} \int_{0}^{s} e^{-p_{2}(s-r)} \int_{0}^{r} \frac{(r-m)^{\varrho-2}}{\Gamma(\varrho-1)} \hat{\vartheta}(m) \mathrm{d} m \mathrm{~d} r \\
& +\frac{1-e^{-p_{2} s}+\left(p_{2}^{2}-p_{2}\right) s}{p_{1}\left(\tilde{\Omega}_{2}-p_{2} \Delta^{*}\right)}\left[\int_{0}^{1} e^{-p_{2}(1-r)} \int_{0}^{r} \frac{(r-m)^{\varrho-2}}{\Gamma(\varrho-1)} \hat{\vartheta}(m) \mathrm{d} m \mathrm{~d} r\right. \\
& \left.+\int_{0}^{p} \frac{(p-r)^{\xi-1}}{\Gamma(\xi)} \int_{0}^{r} e^{-p_{2}(r-\tau)} \int_{0}^{\tau} \frac{(\tau-m)^{\varrho-2}}{\Gamma(\varrho-1)} \hat{\vartheta}(m) \mathrm{d} m \mathrm{~d} \tau \mathrm{d} r\right]
\end{aligned}
$$

for all $s \in[0,1]$. Let $s_{1}, s_{2} \in[0,1]$ be such that $s_{1}<s_{2}$. Then we have

$$
\begin{aligned}
\left|v\left(s_{2}\right)-v\left(s_{1}\right)\right| \leq & \mid \frac{1}{p_{1}} \int_{0}^{s_{2}} e^{-p_{2}\left(s_{2}-r\right)} \int_{0}^{r} \frac{(r-m)^{\varrho-2}}{\Gamma(\varrho-1)} \hat{\vartheta}(m) \mathrm{d} m \mathrm{~d} r \\
& -\frac{1}{p_{1}} \int_{0}^{s_{1}} e^{-p_{2}\left(s_{1}-r\right)} \int_{0}^{r} \frac{(r-m)^{\varrho-2}}{\Gamma(\varrho-1)} \hat{\vartheta}(m) \mathrm{d} m \mathrm{~d} r \mid
\end{aligned}
$$




$$
\begin{aligned}
& +\frac{\left(e^{-p_{2} s_{1}}-e^{-p_{2} s_{2}}\right)+\left|p_{2}^{2}-p_{2}\right|\left(s_{2}-s_{1}\right)}{p_{1}\left|\tilde{\Omega}_{2}-p_{2} \Delta^{*}\right|} \\
& \times\left[\int_{0}^{1} e^{-p_{2}(1-r)} \int_{0}^{r} \frac{(r-m)^{\varrho-2}}{\Gamma(\varrho-1)}|\hat{\vartheta}(m)| \mathrm{d} m \mathrm{~d} r\right. \\
& \left.+\int_{0}^{p} \frac{(p-r)^{\xi-1}}{\Gamma(\xi)} \int_{0}^{r} e^{-p_{2}(r-\tau)} \int_{0}^{\tau} \frac{(\tau-m)^{\varrho-2}}{\Gamma(\varrho-1)}|\hat{\vartheta}(m)| \mathrm{d} m \mathrm{~d} \tau \mathrm{d} r\right] .
\end{aligned}
$$

Thus we observe that the limit of the right-hand side is zero without considering $w \in \mathcal{V}_{\tilde{\varepsilon}}$ as $s_{1} \rightarrow s_{2}$. Therefore by the Arzelà-Ascoli theorem we conclude that $\Phi_{2}^{*}: \mathcal{C}_{\mathbb{R}}([0,1]) \rightarrow$ $\mathcal{P}\left(\mathcal{C}_{\mathbb{R}}([0,1])\right.$ is completely continuous. We further prove that $\Phi_{2}^{*}$ has a closed graph, which implies the upper semicontinuity of this operator. Let $w_{n} \in \mathcal{V}_{\tilde{\varepsilon}}$ and $v_{n} \in \Phi_{2}^{*} w_{n}$ be such that $w_{n} \rightarrow w^{*}$ and $v_{n} \rightarrow v^{*}$. We show the inclusion $v^{*} \in \Phi_{2}^{*} w^{*}$. For each index $n \geq 1$ and $v_{n} \in \Phi_{2}^{*} w_{n}$, we select $\hat{\vartheta}_{n} \in(\mathcal{S E L})_{\mathcal{S}, w_{n}}$ such that

$$
\begin{aligned}
v_{n}(s)= & \frac{1}{p_{1}} \int_{0}^{s} e^{-p_{2}(s-r)} \int_{0}^{r} \frac{(r-m)^{\varrho-2}}{\Gamma(\varrho-1)} \hat{\vartheta}_{n}(m) \mathrm{d} m \mathrm{~d} r \\
& +\frac{1-e^{-p_{2} s}+\left(p_{2}^{2}-p_{2}\right) s}{p_{1}\left(\tilde{\Omega}_{2}-p_{2} \Delta^{*}\right)}\left[\int_{0}^{1} e^{-p_{2}(1-r)} \int_{0}^{r} \frac{(r-m)^{\varrho-2}}{\Gamma(\varrho-1)} \hat{\vartheta}_{n}(m) \mathrm{d} m \mathrm{~d} r\right. \\
& \left.+\int_{0}^{p} \frac{(p-r)^{\xi-1}}{\Gamma(\xi)} \int_{0}^{r} e^{-p_{2}(r-\tau)} \int_{0}^{\tau} \frac{(\tau-m)^{\varrho-2}}{\Gamma(\varrho-1)} \hat{\vartheta}_{n}(m) \mathrm{d} m \mathrm{~d} \tau \mathrm{d} r\right]
\end{aligned}
$$

for all $s \in[0,1]$. We need to prove the existence of a function $\hat{\vartheta}^{*} \in(\mathcal{S E L})_{\mathcal{S}, w^{*}}$ such that

$$
\begin{aligned}
\nu^{*}(s)= & \frac{1}{p_{1}} \int_{0}^{s} e^{-p_{2}(s-r)} \int_{0}^{r} \frac{(r-m)^{\varrho-2}}{\Gamma(\varrho-1)} \hat{\vartheta}^{*}(m) \mathrm{d} m \mathrm{~d} r \\
& +\frac{1-e^{-p_{2} s}+\left(p_{2}^{2}-p_{2}\right) s}{p_{1}\left(\tilde{\Omega}_{2}-p_{2} \Delta^{*}\right)}\left[\int_{0}^{1} e^{-p_{2}(1-r)} \int_{0}^{r} \frac{(r-m)^{\varrho-2}}{\Gamma(\varrho-1)} \hat{\vartheta}^{*}(m) \mathrm{d} m \mathrm{~d} r\right. \\
& \left.+\int_{0}^{p} \frac{(p-r)^{\xi-1}}{\Gamma(\xi)} \int_{0}^{r} e^{-p_{2}(r-\tau)} \int_{0}^{\tau} \frac{(\tau-m)^{\varrho-2}}{\Gamma(\varrho-1)} \hat{\vartheta}^{*}(m) \mathrm{d} m \mathrm{~d} \tau \mathrm{d} r\right]
\end{aligned}
$$

for all $s \in[0,1]$. To arrive at the desired conclusion, we define the continuous linear map $\Xi: \mathcal{L}_{\mathbb{R}}^{1}([0,1]) \rightarrow \mathcal{W}=\mathcal{C}_{\mathbb{R}}([0,1])$ as

$$
\begin{aligned}
\Xi(\hat{\vartheta})(s)= & w(s) \\
= & \frac{1}{p_{1}} \int_{0}^{s} e^{-p_{2}(s-r)} \int_{0}^{r} \frac{(r-m)^{\varrho-2}}{\Gamma(\varrho-1)} \hat{\vartheta}(m) \mathrm{d} m \mathrm{~d} r \\
& +\frac{1-e^{-p_{2} s}+\left(p_{2}^{2}-p_{2}\right) s}{p_{1}\left(\tilde{\Omega}_{2}-p_{2} \Delta^{*}\right)}\left[\int_{0}^{1} e^{-p_{2}(1-r)} \int_{0}^{r} \frac{(r-m)^{\varrho-2}}{\Gamma(\varrho-1)} \hat{\vartheta}(m) \mathrm{d} m \mathrm{~d} r\right. \\
& \left.+\int_{0}^{p} \frac{(p-r)^{\xi-1}}{\Gamma(\xi)} \int_{0}^{r} e^{-p_{2}(r-\tau)} \int_{0}^{\tau} \frac{(\tau-m)^{\varrho-2}}{\Gamma(\varrho-1)} \hat{\vartheta}(m) \mathrm{d} m \mathrm{~d} \tau \mathrm{d} r\right]
\end{aligned}
$$

for all $s \in[0,1]$. We can directly verify that

$$
\begin{aligned}
& \left\|v_{n}(s)-v^{*}(s)\right\| \\
& \quad=\| \frac{1}{p_{1}} \int_{0}^{s} e^{-p_{2}(s-r)} \int_{0}^{r} \frac{(r-m)^{\varrho-2}}{\Gamma(\varrho-1)}\left(\hat{\vartheta}_{n}(m)-\hat{\vartheta}^{*}(m)\right) \mathrm{d} m \mathrm{~d} r
\end{aligned}
$$




$$
\begin{aligned}
& +\frac{1-e^{-p_{2} s}+\left(p_{2}^{2}-p_{2}\right) s}{p_{1}\left(\tilde{\Omega}_{2}-p_{2} \Delta^{*}\right)}\left[\int_{0}^{1} e^{-p_{2}(1-r)} \int_{0}^{r} \frac{(r-m)^{\varrho-2}}{\Gamma(\varrho-1)}\left(\hat{\vartheta}_{n}(m)-\hat{\vartheta}^{*}(m)\right) \mathrm{d} m \mathrm{~d} r\right. \\
& \left.+\int_{0}^{p} \frac{(p-r)^{\xi-1}}{\Gamma(\xi)} \int_{0}^{r} e^{-p_{2}(r-\tau)} \int_{0}^{\tau} \frac{(\tau-m)^{\varrho-2}}{\Gamma(\varrho-1)}\left(\hat{\vartheta}_{n}(m)-\hat{\vartheta}^{*}(m)\right) \mathrm{d} m \mathrm{~d} \tau \mathrm{d} r\right] \| \rightarrow 0 .
\end{aligned}
$$

Hence by Theorem 2, $\Xi \circ(\mathcal{S E L})_{\mathcal{S}}$ has a closed graph. Since $v_{n} \in \Xi\left((\mathcal{S E L})_{\mathcal{S}, w_{n}}\right)$ and $w_{n} \rightarrow w^{*}$, there is a function $\hat{\vartheta}^{*} \in(\mathcal{S E} \mathcal{L})_{\mathcal{S}, w^{*}}$ such that

$$
\begin{aligned}
v^{*}(t)= & \frac{1}{p_{1}} \int_{0}^{s} e^{-p_{2}(s-r)} \int_{0}^{r} \frac{(r-m)^{\varrho-2}}{\Gamma(\varrho-1)} \hat{\vartheta}^{*}(m) \mathrm{d} m \mathrm{~d} r \\
& +\frac{1-e^{-p_{2} s}+\left(p_{2}^{2}-p_{2}\right) s}{p_{1}\left(\tilde{\Omega}_{2}-p_{2} \Delta^{*}\right)}\left[\int_{0}^{1} e^{-p_{2}(1-r)} \int_{0}^{r} \frac{(r-m)^{\varrho-2}}{\Gamma(\varrho-1)} \hat{\vartheta}^{*}(m) \mathrm{d} m \mathrm{~d} r\right. \\
& \left.+\int_{0}^{p} \frac{(p-r)^{\xi-1}}{\Gamma(\xi)} \int_{0}^{r} e^{-p_{2}(r-\tau)} \int_{0}^{\tau} \frac{(\tau-m)^{\varrho-2}}{\Gamma(\varrho-1)} \hat{\vartheta}^{*}(m) \mathrm{d} m \mathrm{~d} \tau \mathrm{d} r\right]
\end{aligned}
$$

for all $s \in[0,1]$. Thus $v^{*} \in \Phi_{2}^{*} w^{*}$, and so $\Phi_{2}^{*}$ has a closed graph. This implies that $\Phi_{2}^{*}$ is upper semicontinuous. On the other hand, note that the operator $\Phi_{2}^{*}$ has compact values. Hence $\Phi_{2}^{*}$ is a compact and upper semicontinuous. In the next step, in addition to hypothesis (Hyp3), by a similar argument we get

$$
\begin{aligned}
\hat{\Delta}= & \left\|\Phi_{2}^{*}(\mathcal{W})\right\| \\
= & \sup _{s \in[0,1]}\left\{\left|\Phi_{2}^{*} w\right|: w \in \mathcal{W}\right\} \\
\leq & {\left[\frac{\left(1-e^{-p_{2}}\right)}{p_{1} p_{2} \Gamma(\varrho)}+\frac{\left|1-e^{-p_{2}}\right|+\left|p_{2}^{2}-p_{2}\right|}{p_{1}\left|\tilde{\Omega}_{2}-p_{2} \Delta^{*}\right|}\right.} \\
& \left.\quad \times\left(\frac{\left(1-e^{-p_{2}}\right)}{p_{2} \Gamma(\varrho)}+\frac{p^{\varrho+\xi-1}\left(p_{2} p+e^{-p_{2} p}-1\right)}{p_{2}^{2} \Gamma(\varrho) \Gamma(\xi)}\right)\right]\|\theta\|_{\mathcal{L}^{1}} \\
= & M\|\theta\|_{\mathcal{L}^{1}},
\end{aligned}
$$

and so $\hat{\Delta} \leq M\|\theta\|_{\mathcal{L}^{1}}$. Hence $v^{*}\left(1+\frac{1}{\Gamma(\gamma+1)}\right) \hat{\Delta} \leq v^{*}\left(1+\frac{1}{\Gamma(\gamma+1)}\right) M\|\theta\|_{\mathcal{L}^{1}}<\frac{1}{2}$. Set $l^{*}=$ $\nu^{*}\left(1+\frac{1}{\Gamma(\gamma+1)}\right)$. It is clear that $l^{*} \hat{\Delta}<\frac{1}{2}$. We see that all three assumptions of Theorem 2 are satisfied for the operators $\Phi_{1}^{*}$ and $\Phi_{2}^{*}$. Now we only need to show that one of conditions (a) or (b) is valid. We claim that the invalid condition is (b). To observe this, by Theorem 2 and hypothesis (Hyp4) we may assume that $w$ is an arbitrary element belonging to $\mathcal{O}^{*}$ with $\|w\|=q$. Obviously, $\alpha_{0} w(s) \in \Phi_{1}^{*} w(s) \Phi_{2}^{*} w(s)$ for any $\alpha_{0}>1$. We select the corresponding function $\hat{\vartheta} \in(\mathcal{S E L})_{\mathcal{S}, w}$. Then for each $\alpha_{0}>1$, we have

$$
\begin{aligned}
w(s)= & \frac{1}{\alpha_{0}} \zeta\left(s, w_{0}(s),{ }^{R} I_{0^{+}}^{\gamma} w_{0}(s)\right)\left(\frac{1}{p_{1}} \int_{0}^{s} e^{-p_{2}(s-r)} \int_{0}^{r} \frac{(r-m)^{\varrho-2}}{\Gamma(\varrho-1)} \hat{\vartheta}^{*}(m) \mathrm{d} m \mathrm{~d} r\right. \\
& +\frac{1-e^{-p_{2} s}+\left(p_{2}^{2}-p_{2}\right) s}{p_{1}\left(\tilde{\Omega}_{2}-p_{2} \Delta^{*}\right)}\left[\int_{0}^{1} e^{-p_{2}(1-r)} \int_{0}^{r} \frac{(r-m)^{\varrho-2}}{\Gamma(\varrho-1)} \hat{\vartheta}^{*}(m) \mathrm{d} m \mathrm{~d} r\right. \\
& \left.\left.+\int_{0}^{p} \frac{(p-r)^{\xi-1}}{\Gamma(\xi)} \int_{0}^{r} e^{-p_{2}(r-\tau)} \int_{0}^{\tau} \frac{(\tau-m)^{\varrho-2}}{\Gamma(\varrho-1)} \hat{\vartheta}^{*}(m) \mathrm{d} m \mathrm{~d} \tau \mathrm{d} r\right]\right)
\end{aligned}
$$


for all $s \in[0,1]$. Thus we can write

$$
\begin{aligned}
|w(s)|= & \frac{1}{\alpha_{0}}\left|\zeta\left(s, w(s),{ }^{R} I_{0^{+}}^{\gamma} w(s)\right)\right| \\
& \times\left(\frac{1}{p_{1}} \int_{0}^{s} e^{-p_{2}(s-r)} \int_{0}^{r} \frac{(r-m)^{\varrho-2}}{\Gamma(\varrho-1)}|\hat{\vartheta}(m)| \mathrm{d} m \mathrm{~d} r\right. \\
& +\frac{\left|1-e^{-p_{2} s}\right|+\left|p_{2}^{2}-p_{2}\right| s}{p_{1}\left|\tilde{\Omega}_{2}-p_{2} \Delta^{*}\right|}\left[\int_{0}^{1} e^{-p_{2}(1-r)} \int_{0}^{r} \frac{(r-m)^{\varrho-2}}{\Gamma(\varrho-1)}|\hat{\vartheta}(m)| \mathrm{d} m \mathrm{~d} r\right. \\
& \left.\left.+\int_{0}^{p} \frac{(p-r)^{\xi-1}}{\Gamma(\xi)} \int_{0}^{r} e^{-p_{2}(r-\tau)} \int_{0}^{\tau} \frac{(\tau-m)^{\varrho-2}}{\Gamma(\varrho-1)}|\hat{\vartheta}(m)| \mathrm{d} m \mathrm{~d} \tau \mathrm{d} r\right]\right) \\
= & {\left[\zeta\left(s, w(s),{ }^{R} I_{0^{+}}^{\gamma} w(s)\right)-\zeta(s, 0,0)|+| \zeta(0,0,0) \mid\right] } \\
& \times\left(\frac{1}{p_{1}} \int_{0}^{s} e^{-p_{2}(s-r)} \int_{0}^{r} \frac{(r-m)^{\varrho-2}}{\Gamma(\varrho-1)}|\hat{\vartheta}(m)| \mathrm{d} m \mathrm{~d} r\right. \\
& +\frac{\left|1-e^{-p_{2} s}\right|+\left|p_{2}^{2}-p_{2}\right| s}{p_{1}\left|\tilde{\Omega}_{2}-p_{2} \Delta^{*}\right|}\left[\int_{0}^{1} e^{-p_{2}(1-r)} \int_{0}^{r} \frac{(r-m)^{\varrho-2}}{\Gamma(\varrho-1)}|\hat{\vartheta}(m)| \mathrm{d} m \mathrm{~d} r\right. \\
& \left.\left.+\int_{0}^{p} \frac{(p-r)^{\xi-1}}{\Gamma(\xi)} \int_{0}^{r} e^{-p_{2}(r-\tau)} \int_{0}^{\tau} \frac{(\tau-m)^{\varrho-2}}{\Gamma(\varrho-1)}|\hat{\vartheta}(m)| \mathrm{d} m \mathrm{~d} \tau \mathrm{d} r\right]\right) \\
\leq & {\left[v^{*}\left(1+\frac{1}{\Gamma(\gamma+1)}\right)\|w\|+\zeta^{*}\right] } \\
& \times\left(\frac{1}{p_{1}} \int_{0}^{s} e^{-p_{2}(s-r)} \int_{0}^{r} \frac{(r-m)^{\varrho-2}}{\Gamma(\varrho-1)} \theta(m) \mathrm{d} m \mathrm{~d} r\right. \\
& +\frac{\left|1-e^{-p_{2}}\right|+\left|p_{2}^{2}-p_{2}\right|}{p_{1}\left|\tilde{\Omega}_{2}-p_{2} \Delta^{*}\right|}\left[\int_{0}^{1} e^{-p_{2}(1-r)} \int_{0}^{r} \frac{(r-m)^{\varrho-2}}{\Gamma(\varrho-1)} \theta(m) \mathrm{d} m \mathrm{~d} r\right. \\
& \left.\left.+\int_{0}^{p} \frac{(p-r)^{\xi-1}}{\Gamma(\xi)} \int_{0}^{r} e^{-p_{2}(r-\tau)} \int_{0}^{\tau} \frac{(\tau-m)^{\varrho-2}}{\Gamma(\varrho-1)} \theta(m) \mathrm{d} m \mathrm{~d} \tau \mathrm{d} r\right]\right) \\
& {\left[v^{*}\left(1+\frac{1}{\Gamma(\gamma+1)}\right)\|w\|+\zeta^{*}\right] M\|\theta\| \mathcal{L}^{1} }
\end{aligned}
$$

for all $s \in[0,1]$. Hence $q \leq \frac{\zeta^{*} M\|\theta\|_{\mathcal{L}^{1}}}{1-v^{*}\left(1+\frac{1}{\Gamma(\gamma+1)}\right) M\|\theta\|_{\mathcal{L}^{1}}}$. According to (13), the impossibility of condition (b) of Theorem 2 follows. Consequently, we have $w \in \Phi_{1}^{*} w \Phi_{2}^{*} w$. Hence the existence of a fixed point for the operator $\mathcal{G}$ is proved, and thus the sequential hybrid inclusion BVP (1)-(2) has a solution. This completes the proof.

In this position, we continue our process to reach the existence results for the sequential nonhybrid inclusion BVP (3)-(4) by using two new theoretical theorems.

Definition 9 A function $w \in \mathcal{A C}_{\mathbb{R}}([0,1])$ is a solution of the sequential inclusion BVP (3)-(4) if there is an integrable function $\hat{\vartheta} \in \mathcal{L}_{\mathbb{R}}^{1}([0,1])$ with $\hat{\vartheta} \in \mathcal{S}(s, w(s))$ for almost all $s \in[0,1]$ satisfying the three-point integro-derivative boundary conditions

$$
\begin{aligned}
& w(0)=0, \quad w^{\prime}(0)+w^{\prime \prime}(0)=0, \\
& w(1)+{ }^{R} I_{0^{+}}^{\xi} w(p)=0,
\end{aligned}
$$


and

$$
\begin{aligned}
w(s)= & \frac{1}{p_{1}} \int_{0}^{s} e^{-p_{2}(s-r)} \int_{0}^{r} \frac{(r-m)^{\varrho-2}}{\Gamma(\varrho-1)} \hat{\vartheta}(m) \mathrm{d} m \mathrm{~d} r \\
& +\frac{1-e^{-p_{2} s}+\left(p_{2}^{2}-p_{2}\right) s}{p_{1}\left(\tilde{\Omega}_{2}-p_{2} \Delta^{*}\right)}\left[\int_{0}^{1} e^{-p_{2}(1-r)} \int_{0}^{r} \frac{(r-m)^{\varrho-2}}{\Gamma(\varrho-1)} \hat{\vartheta}(m) \mathrm{d} m \mathrm{~d} r\right. \\
& \left.+\int_{0}^{p} \frac{(p-r)^{\xi-1}}{\Gamma(\xi)} \int_{0}^{r} e^{-p_{2}(r-\tau)} \int_{0}^{\tau} \frac{(\tau-m)^{\varrho-2}}{\Gamma(\varrho-1)} \hat{\vartheta}(m) \mathrm{d} m \mathrm{~d} \tau \mathrm{d} r\right]
\end{aligned}
$$

for any $s \in[0,1]$.

Now, for each $w \in \mathcal{W}$, we introduce the following family of selections of $\mathcal{S}$ :

$$
\left.(\mathcal{S E} \mathcal{L})_{\mathcal{S}, w}=\left\{\hat{\vartheta} \in L^{1}([0,1]): \hat{\vartheta}(s) \in \mathcal{S}(s, w(s))\right) \text { for almost all } s \in[0,1]\right\} .
$$

Define the operator $\mathcal{K}: \mathcal{W} \rightarrow \mathcal{P}(\mathcal{W})$ by

$$
\mathcal{K}(w)=\left\{z \in \mathcal{W}: \text { there is } \hat{\vartheta} \in(\mathcal{S E} \mathcal{L})_{\mathcal{S}, w} \text { such that } z(s)=h(s) \text { for any } s \in[0,1]\right\}
$$

where

$$
\begin{aligned}
h(s)= & \frac{1}{p_{1}} \int_{0}^{s} e^{-p_{2}(s-r)} \int_{0}^{r} \frac{(r-m)^{\varrho-2}}{\Gamma(\varrho-1)} \hat{\vartheta}(m) \mathrm{d} m \mathrm{~d} r \\
& +\frac{1-e^{-p_{2} s}+\left(p_{2}^{2}-p_{2}\right) s}{p_{1}\left(\tilde{\Omega}_{2}-p_{2} \Delta^{*}\right)}\left[\int_{0}^{1} e^{-p_{2}(1-r)} \int_{0}^{r} \frac{(r-m)^{\varrho-2}}{\Gamma(\varrho-1)} \hat{\vartheta}(m) \mathrm{d} m \mathrm{~d} r\right. \\
& \left.+\int_{0}^{p} \frac{(p-r)^{\xi-1}}{\Gamma(\xi)} \int_{0}^{r} e^{-p_{2}(r-\tau)} \int_{0}^{\tau} \frac{(\tau-m)^{\varrho-2}}{\Gamma(\varrho-1)} \hat{\vartheta}(m) \mathrm{d} m \mathrm{~d} \tau \mathrm{d} r\right] .
\end{aligned}
$$

Theorem 10 Let $\mathcal{S}:[0,1] \times \mathcal{W} \rightarrow \mathcal{P}_{\mathrm{cmp}}(\mathcal{W})$ be a compact set-valued map. Assume that:

(Hyp5) The bounded operator $\mathcal{S}$ is integrable, and $\mathcal{S}(\cdot, w):[0,1] \rightarrow \mathcal{P}_{\mathrm{cmp}}(\mathcal{W})$ is measurable for all $w \in \mathcal{W}$;

(Hyp6) There are $\psi \in \Psi$ and $\sigma \in \mathcal{C}_{\mathbb{R} \geq 0}([0,1])$ such that

$$
\operatorname{PH}_{d_{\mathcal{W}}}\left(\mathcal{S}(s, w), \mathcal{S}\left(s, w^{\prime}\right)\right) \leq \sigma(s) \psi\left(\left|w-w^{\prime}\right|\right) \frac{1}{M\|\sigma\|}
$$

for all $s \in[0,1]$ and $w, w^{\prime} \in \mathcal{W}$, where $\sup _{s \in[0,1]}|\sigma(s)|=\|\sigma\|$, and the constant $M$ is given by (14);

(Hyp7) There is a function $\tilde{\zeta}: \mathbb{R} \times \mathbb{R} \rightarrow \mathbb{R}$ such that $\tilde{\zeta}\left(w, w^{\prime}\right) \geq 0$ for all $w, w^{\prime} \in \mathcal{W}$;

(Hyp8) If the sequence $\left\{w_{n}\right\}_{n \geq 1}$ in $\mathcal{W}$ converges to $w$ and $\tilde{\zeta}\left(w_{n}(s), w_{n+1}(s)\right) \geq 0$ for all $s \in[0,1]$ and $n \geq 1$, then there is a subsequence $\left\{w_{n_{l}}\right\}_{l \geq 1}$ of $\left\{w_{n}\right\}$ such that $\tilde{\zeta}\left(w_{n_{l}}(s), w(s)\right) \geq 0$ for all $s \in[0,1]$ and $l \geq 1$;

(Hyp9) There are two elements $w_{0} \in \mathcal{W}$ and $z \in \mathcal{K}\left(w_{0}\right)$ such that $\tilde{\zeta}\left(w_{0}(s), z(s)\right) \geq 0$ for all $s \in[0,1]$, where $\mathcal{K}: \mathcal{W} \rightarrow \mathcal{P}(\mathcal{W})$ is the operator defined by $(15) ;$

(Hyp10) For all $w \in \mathcal{W}$ and $z \in \mathcal{K}(w)$ with $\tilde{\zeta}(w(s), z(s)) \geq 0$, there is $h \in \mathcal{K}(w)$ such that $\tilde{\zeta}(z(s), h(s)) \geq 0$ for all $s \in[0,1]$.

Then the three-point sequential inclusion BVP (3)-(4) has a solution. 
Proof In a similar manner, each fixed point of the operator $\mathcal{K}: \mathcal{W} \rightarrow \mathcal{P}(\mathcal{W})$ given by $(15)$ is a solution of the sequential inclusion BVP (3)-(4). Due to assumption (Hyp5), the measurability of the set-valued map $s \mapsto \mathcal{S}(s, w(s))$ is clear, and so it is closed-valued for all $w \in \mathcal{W}$. Hence $\mathcal{S}$ has a measurable selection, and $(\mathcal{S E} \mathcal{L})_{\mathcal{S}, w} \neq \emptyset$. Now we want to prove that $\mathcal{K}(w)$ is a closed subset of $\mathcal{W}$ for all $w \in \mathcal{W}$. To this end, we consider a sequence $\left\{w_{n}\right\}_{n \geq 1}$ of $\mathcal{K}(w)$ such that $w_{n} \rightarrow w$. For each $n$, choose $\hat{\vartheta}_{n} \in(\mathcal{S E L})_{\mathcal{S}, w}$ such that

$$
\begin{aligned}
w_{n}(s)= & \frac{1}{p_{1}} \int_{0}^{s} e^{-p_{2}(s-r)} \int_{0}^{r} \frac{(r-m)^{\varrho-2}}{\Gamma(\varrho-1)} \hat{\vartheta}_{n}(m) \mathrm{d} m \mathrm{~d} r \\
& +\frac{1-e^{-p_{2} s}+\left(p_{2}^{2}-p_{2}\right) s}{p_{1}\left(\tilde{\Omega}_{2}-p_{2} \Delta^{*}\right)}\left[\int_{0}^{1} e^{-p_{2}(1-r)} \int_{0}^{r} \frac{(r-m)^{\varrho-2}}{\Gamma(\varrho-1)} \hat{\vartheta}_{n}(m) \mathrm{d} m \mathrm{~d} r\right. \\
& \left.+\int_{0}^{p} \frac{(p-r)^{\xi-1}}{\Gamma(\xi)} \int_{0}^{r} e^{-p_{2}(r-\tau)} \int_{0}^{\tau} \frac{(\tau-m)^{\varrho-2}}{\Gamma(\varrho-1)} \hat{\vartheta}_{n}(m) \mathrm{d} m \mathrm{~d} \tau \mathrm{d} r\right]
\end{aligned}
$$

for almost all $s \in[0,1]$. Because of the compactness of the set-valued map $\mathcal{S}$, we may pass to a convergent subsequence $\left\{\hat{\vartheta}_{n}\right\}_{n \geq 1}$ (if necessary), which tends to some $\hat{\vartheta} \in \mathcal{L}^{1}([0,1])$. Therefore we have $\hat{\vartheta} \in(\mathcal{S E L})_{\mathcal{S}, w}$, and so

$$
\begin{aligned}
\lim _{n \rightarrow \infty} w_{n}(s)= & \frac{1}{p_{1}} \int_{0}^{s} e^{-p_{2}(s-r)} \int_{0}^{r} \frac{(r-m)^{\varrho-2}}{\Gamma(\varrho-1)} \hat{\vartheta}(m) \mathrm{d} m \mathrm{~d} r \\
& +\frac{1-e^{-p_{2} s}+\left(p_{2}^{2}-p_{2}\right) s}{p_{1}\left(\tilde{\Omega}_{2}-p_{2} \Delta^{*}\right)}\left[\int_{0}^{1} e^{-p_{2}(1-r)} \int_{0}^{r} \frac{(r-m)^{\varrho-2}}{\Gamma(\varrho-1)} \hat{\vartheta}(m) \mathrm{d} m \mathrm{~d} r\right. \\
& \left.+\int_{0}^{p} \frac{(p-r)^{\xi-1}}{\Gamma(\xi)} \int_{0}^{r} e^{-p_{2}(r-\tau)} \int_{0}^{\tau} \frac{(\tau-m)^{\varrho-2}}{\Gamma(\varrho-1)} \hat{\vartheta}(m) \mathrm{d} m \mathrm{~d} \tau \mathrm{d} r\right]=w(s)
\end{aligned}
$$

for all $s \in[0,1]$. Hence $w \in \mathcal{K}(w)$, and so $\mathcal{K}$ has closed values. By the assumptions of the theorem we know that $\mathcal{S}$ is a compact set-valued map. Thus we can easily check that the set $\mathcal{K}(w)$ is bounded for all $w \in \mathcal{W}$. In this position, we are going to prove that the operator $\mathcal{K}$ is an $\alpha$ - $\psi$-contraction. To see this, define the nonnegative function $\alpha: \mathcal{W} \times \mathcal{W} \rightarrow[0, \infty)$ by $\alpha\left(w, w^{\prime}\right)=1$ if $\tilde{\zeta}\left(w(s), w^{\prime}(s)\right) \geq 0$ and $\alpha\left(w, w^{\prime}\right)=0$ otherwise. Let $w, w^{\prime} \in \mathcal{W}$ and $z_{1} \in \mathcal{K}\left(w^{\prime}\right)$. Choose $\hat{\vartheta}_{1} \in(\mathcal{S E L})_{\mathcal{S}, w^{\prime}}$ such that

$$
\begin{aligned}
z_{1}(s)= & \frac{1}{p_{1}} \int_{0}^{s} e^{-p_{2}(s-r)} \int_{0}^{r} \frac{(r-m)^{\varrho-2}}{\Gamma(\varrho-1)} \hat{\vartheta}_{1}(m) \mathrm{d} m \mathrm{~d} r \\
& +\frac{1-e^{-p_{2} s}+\left(p_{2}^{2}-p_{2}\right) s}{p_{1}\left(\tilde{\Omega}_{2}-p_{2} \Delta^{*}\right)}\left[\int_{0}^{1} e^{-p_{2}(1-r)} \int_{0}^{r} \frac{(r-m)^{\varrho-2}}{\Gamma(\varrho-1)} \hat{\vartheta}_{1}(m) \mathrm{d} m \mathrm{~d} r\right. \\
& \left.+\int_{0}^{p} \frac{(p-r)^{\xi-1}}{\Gamma(\xi)} \int_{0}^{r} e^{-p_{2}(r-\tau)} \int_{0}^{\tau} \frac{(\tau-m)^{\varrho-2}}{\Gamma(\varrho-1)} \hat{\vartheta}_{1}(m) \mathrm{d} m \mathrm{~d} \tau \mathrm{d} r\right]
\end{aligned}
$$

for all $s \in[0,1]$. Under condition (16), we get

$$
\operatorname{PH}_{d_{\mathcal{W}}}\left(\mathcal{S}(s, w(s)), \mathcal{S}\left(s, w^{\prime}(s)\right)\right) \leq \sigma(s) \psi\left(\left|w(s)-w^{\prime}(s)\right|\right) \frac{1}{M\|\sigma\|}
$$

for all $w, w^{\prime} \in \mathcal{W}$ such that $\tilde{\zeta}\left(w(s), w^{\prime}(s)\right) \geq 0$ for $s \in[0,1]$. Therefore there is $h \in \mathcal{S}(s, w(s))$ such that $\left|\hat{\vartheta}_{1}(s)-h\right| \leq \sigma(s) \psi\left(\left|w(s)-w^{\prime}(s)\right|\right) \frac{1}{M\|\sigma\|}$. We further introduce the new set-valued 
map $\mathcal{B}^{*}:[0,1] \rightarrow \mathcal{P}(\mathcal{W})$ defined by

$$
\mathcal{B}^{*}(s)=\left\{h \in \mathcal{W}:\left|\hat{\vartheta}_{1}(s)-h\right| \leq \sigma(s) \psi\left(\left|w(s)-w^{\prime}(s)\right|\right) \frac{1}{M\|\sigma\|}\right\}
$$

for $s \in[0,1]$. Since $\hat{\vartheta}_{1}$ and $\varpi=\sigma \psi\left(\left|w-w^{\prime}\right|\right) \frac{1}{M\|\sigma\|}$ are measurable, the intersection of two set-valued maps $\mathcal{B}^{*}(\cdot) \cap \mathcal{S}(\cdot, w(\cdot))$ is measurable. In this direction, we choose $\hat{\vartheta}_{2}$ belonging to $\mathcal{S}(s, w(s))$ such that $\left|\hat{\vartheta}_{1}(s)-\hat{\vartheta}_{2}(s)\right| \leq \sigma(s) \psi\left(\left|w(s)-w^{\prime}(s)\right|\right) \frac{1}{M\|\sigma\|}$ for all $s \in[0,1]$. Define the element $z_{2} \in \mathcal{K}(w)$ by

$$
\begin{aligned}
z_{2}(s)= & \frac{1}{p_{1}} \int_{0}^{s} e^{-p_{2}(s-r)} \int_{0}^{r} \frac{(r-m)^{\varrho-2}}{\Gamma(\varrho-1)} \hat{\vartheta}_{2}(m) \mathrm{d} m \mathrm{~d} r \\
& +\frac{1-e^{-p_{2} s}+\left(p_{2}^{2}-p_{2}\right) s}{p_{1}\left(\tilde{\Omega}_{2}-p_{2} \Delta^{*}\right)}\left[\int_{0}^{1} e^{-p_{2}(1-r)} \int_{0}^{r} \frac{(r-m)^{\varrho-2}}{\Gamma(\varrho-1)} \hat{\vartheta}_{2}(m) \mathrm{d} m \mathrm{~d} r\right. \\
& \left.+\int_{0}^{p} \frac{(p-r)^{\xi-1}}{\Gamma(\xi)} \int_{0}^{r} e^{-p_{2}(r-\tau)} \int_{0}^{\tau} \frac{(\tau-m)^{\varrho-2}}{\Gamma(\varrho-1)} \hat{\vartheta}_{2}(m) \mathrm{d} m \mathrm{~d} \tau \mathrm{d} r\right]
\end{aligned}
$$

for $s \in[0,1]$. Then we can compute the following estimate:

$$
\begin{aligned}
\mid z_{1}(s) & -z_{2}(s) \mid \\
\leq & \frac{1}{p_{1}} \int_{0}^{s} e^{-p_{2}(s-r)} \int_{0}^{r} \frac{(r-m)^{\varrho-2}}{\Gamma(\varrho-1)}\left|\hat{\vartheta}_{1}(m)-\hat{\vartheta}_{2}(m)\right| \mathrm{d} m \mathrm{~d} r \\
& +\frac{\left|1-e^{-p_{2} s}\right|+\left|p_{2}^{2}-p_{2}\right| s}{p_{1}\left|\tilde{\Omega}_{2}-p_{2} \Delta^{*}\right|}\left[\int_{0}^{1} e^{-p_{2}(1-r)} \int_{0}^{r} \frac{(r-m)^{\varrho-2}}{\Gamma(\varrho-1)}\left|\hat{\vartheta}_{1}(m)-\hat{\vartheta}_{2}(m)\right| \mathrm{d} m \mathrm{~d} r\right. \\
& \left.+\int_{0}^{p} \frac{(p-r)^{\xi-1}}{\Gamma(\xi)} \int_{0}^{r} e^{-p_{2}(r-\tau)} \int_{0}^{\tau} \frac{(\tau-m)^{\varrho-2}}{\Gamma(\varrho-1)}\left|\hat{\vartheta}_{1}(m)-\hat{\vartheta}_{2}(m)\right| \mathrm{d} m \mathrm{~d} \tau \mathrm{d} r\right] \\
\leq & {\left[\frac{\left(1-e^{\left.-p_{2}\right)}\right.}{p_{1} p_{2} \Gamma(\varrho)}+\frac{\left|1-e^{-p_{2}}\right|+\left|p_{2}^{2}-p_{2}\right|}{p_{1}\left|\tilde{\Omega}_{2}-p_{2} \Delta^{*}\right|}\left(\frac{\left(1-e^{-p_{2}}\right)}{p_{2} \Gamma(\varrho)}+\frac{p^{\varrho+\xi-1}\left(p_{2} p+e^{-p_{2} p}-1\right)}{p_{2}^{2} \Gamma(\varrho) \Gamma(\xi)}\right)\right] } \\
& \times\|\sigma\| \psi\left(\left\|w-w^{\prime}\right\|\right) \frac{1}{M\|\sigma\|}=M\|\sigma\| \psi\left(\left\|w-w^{\prime}\right\|\right) \frac{1}{M\|\sigma\|}=\psi\left(\left\|w-w^{\prime}\right\|\right)
\end{aligned}
$$

for $s \in[0,1]$. Hence $\left\|z_{1}-z_{2}\right\|=\sup _{s \in[0,1]}\left|z_{1}(s)-z_{2}(s)\right| \leq \psi\left(\left\|w-w^{\prime}\right\|\right)$, and so

$$
\alpha\left(w, w^{\prime}\right) \mathrm{PH}_{d_{\mathcal{W}}}\left(\mathcal{K}(w), \mathcal{K}\left(w^{\prime}\right)\right) \leq \psi\left(\left\|w-w^{\prime}\right\|\right)
$$

for all $w, w^{\prime} \in \mathcal{W}$. This shows that the set-valued map $\mathcal{K}$ is an $\alpha$ - $\psi$-contraction. Consider two elements $w \in \mathcal{W}$ and $w^{\prime} \in \mathcal{K}(w)$ with $\alpha\left(w, w^{\prime}\right) \geq 1$. Then $\tilde{\zeta}\left(w(s), w^{\prime}(s)\right) \geq 0$, and so there is a function $h \in \mathcal{K}\left(w^{\prime}\right)$ such that $\tilde{\zeta}\left(w^{\prime}(s), h(s)\right) \geq 0$. Consequently, $\alpha\left(w^{\prime}, h\right) \geq 1$, which means that $\mathcal{K}$ is $\alpha$-admissible. Now assume that $w_{0} \in \mathcal{W}$ and $w^{\prime} \in \mathcal{K}\left(w_{0}\right)$ are such that $\tilde{\zeta}\left(w_{0}(s), w^{\prime}(s)\right) \geq 0$ for all $s$. Then $\alpha\left(w_{0}, w^{\prime}\right) \geq 1$. Consider the sequence $\left\{w_{n}\right\}_{n \geq 1}$ of $\mathcal{W}$ with $w_{n} \rightarrow w$ and $\alpha\left(w_{n}, w_{n+1}\right) \geq 1$ for each $n$. Then we have $\tilde{\zeta}\left(w_{n}(s), w_{n+1}(s)\right) \geq 0$. By assumption (Hyp8) there is a subsequence $\left\{w_{n_{l}}\right\}_{l \geq 1}$ of $\left\{w_{n}\right\}$ such that $\tilde{\zeta}\left(w_{n_{l}}(s), w(s)\right) \geq 0$ for all $s \in[0,1]$. Thus $\alpha\left(w_{n l}, w\right) \geq 1$ for all $l \geq 1$, and so $\mathcal{W}$ has property $\left(\mathcal{C}_{\alpha}\right)$. Now by Theorem 3 the setvalued map $\mathcal{K}$ has a fixed point, which is as a solution of the sequential inclusion BVP (3)-(4). 
Now we prove another existence result under a new condition due to Amini-Harandi [46]. In this direction, we utilize the approximate endpoint property for $\mathcal{K}$ given by (15).

Theorem 11 Let $\mathcal{S}:[0,1] \times \mathcal{W} \rightarrow \mathcal{P}_{\mathrm{cmp}}(\mathcal{W})$ be a compact set-valued map. Assume that:

(Hyp11) The nondecreasing nonnegative function $\psi:[0, \infty) \rightarrow[0, \infty)$ has the upper semicontinuity property such that $\liminf _{s \rightarrow \infty}(s-\psi(s))>0$ and $\psi(s)<s$ for all $s>0$

(Hyp12) The compact bounded operator $\mathcal{S}:[0,1] \times \mathcal{W} \rightarrow \mathcal{P}_{\mathrm{cmp}}(\mathcal{W})$ is integrable, and for each $w \in \mathcal{W}, \mathcal{S}(\cdot, w):[0,1] \rightarrow \mathcal{P}_{\mathrm{cp}}(\mathcal{W})$ is measurable;

(Hyp13) There is a nonnegative function $\delta \in \mathcal{C}_{\mathbb{R} \geq 0}([0,1])$ such that

$$
\operatorname{PH}_{d_{\mathcal{W}}}\left(\mathcal{S}(s, w)-\mathcal{S}\left(s, w^{\prime}\right)\right) \leq \delta(s) \psi\left(\left|w-w^{\prime}\right|\right) \frac{1}{M\|\delta\|}
$$

for all $s \in[0,1]$ and $w, w^{\prime} \in \mathcal{W}$, where $\sup _{s \in[0,1]}|\delta(s)|=\|\delta\|$, and $M$ is defined by (14);

(Hyp14) The operator $\mathcal{K}$ defined by (15) has the approximate endpoint property.

Then the sequential inclusion BVP (3)-(4) has a solution.

Proof First, we prove the existence of an endpoint for the set-valued map $\mathcal{K}: \mathcal{W} \rightarrow \mathcal{P}(\mathcal{W})$. In this way, we must show that the set $\mathcal{K}(w)$ is closed for all $w \in \mathcal{W}$. Since the map $s \mapsto$ $\mathcal{S}(s, w(s))$ is measurable and closed for all $w \in \mathcal{W}, \mathcal{S}$ has a measurable selection, and so $(\mathcal{S E} \mathcal{L})_{\mathcal{S}, w} \neq \emptyset$ for all $w \in \mathcal{W}$. By using a proof similar to that of Theorem 10 , we easily to check that $\mathcal{K}(w)$ is a closed subset of $\mathcal{W}$. Also, we know that the set $\mathcal{K}(w)$ is bounded for all $w \in \mathcal{W}$ due to the compactness of $\mathcal{S}$. Now we prove that $\operatorname{PH}_{d_{\mathcal{W}}}\left(\mathcal{K}(w), \mathcal{K}\left(w^{\prime}\right)\right) \leq \psi\left(\left\|w-w^{\prime}\right\|\right)$ for every two elements of $\mathcal{W}$. To see this, assume that $w, w^{\prime} \in \mathcal{W}$ and $z_{1} \in \mathcal{K}\left(w^{\prime}\right)$. Choose $\hat{\vartheta}_{1} \in(\mathcal{S E} \mathcal{L})_{\mathcal{S}, w^{\prime}}$ such that

$$
\begin{aligned}
z_{1}(s)= & \frac{1}{p_{1}} \int_{0}^{s} e^{-p_{2}(s-r)} \int_{0}^{r} \frac{(r-m)^{\varrho-2}}{\Gamma(\varrho-1)} \hat{\vartheta}_{1}(m) \mathrm{d} m \mathrm{~d} r \\
& +\frac{1-e^{-p_{2} s}+\left(p_{2}^{2}-p_{2}\right) s}{p_{1}\left(\tilde{\Omega}_{2}-p_{2} \Delta^{*}\right)}\left[\int_{0}^{1} e^{-p_{2}(1-r)} \int_{0}^{r} \frac{(r-m)^{\varrho-2}}{\Gamma(\varrho-1)} \hat{\vartheta}_{1}(m) \mathrm{d} m \mathrm{~d} r\right. \\
& \left.+\int_{0}^{p} \frac{(p-r)^{\xi-1}}{\Gamma(\xi)} \int_{0}^{r} e^{-p_{2}(r-\tau)} \int_{0}^{\tau} \frac{(\tau-m)^{\varrho-2}}{\Gamma(\varrho-1)} \hat{\vartheta}_{1}(m) \mathrm{d} m \mathrm{~d} \tau \mathrm{d} r\right]
\end{aligned}
$$

for almost all $s \in[0,1]$. By considering inequality (17) in hypothesis (Hyp13) we get $\operatorname{PH}_{d_{\mathcal{W}}}\left(\mathcal{S}(s, w(s)), \mathcal{S}\left(s, w^{\prime}(s)\right)\right) \leq \delta(s) \psi\left(\left|w(s)-w^{\prime}(s)\right|\right) \frac{1}{M\|\delta\|}$ for all $s \in[0,1]$. Thus there is $h^{*} \in \mathcal{S}(s, w(s))$ such that $\left|\hat{\vartheta}_{1}(s)-h^{*}\right| \leq \delta(s) \psi\left(\left|w(s)-w^{\prime}(s)\right|\right) \frac{1}{M\|\delta\|}$ for all $s \in[0,1]$. Now define the set-valued map $\mathcal{Q}:[0,1] \rightarrow \mathcal{P}(\mathcal{W})$ by

$$
\mathcal{Q}(s)=\left\{h^{*} \in \mathcal{W}:\left|\hat{\vartheta}_{1}(s)-h^{*}\right| \leq \delta(s) \psi\left(\left|w(s)-w^{\prime}(s)\right|\right) \frac{1}{M\|\delta\|}\right\}
$$

We know that $\hat{\vartheta}_{1}$ and $\varpi=\delta \psi\left(\left|w-w^{\prime}\right|\right) \frac{1}{M\|\delta\|}$ are measurable. Hence we can easily deduce that the intersection set-valued map $\mathcal{Q}(\cdot) \cap \mathcal{S}(\cdot, w(\cdot))$ is measurable. Now choose $\hat{\vartheta}_{2}(s) \in$ $\mathcal{S}(s, w(s))$ such that $\left|\hat{\vartheta}_{1}(s)-\hat{\vartheta}_{2}(s)\right| \leq \delta(s) \psi\left(\left|w(s)-w^{\prime}(s)\right|\right) \frac{1}{M\|\delta\|}$ for all $s \in[0,1]$. Choose $z_{2} \in$ 
$\mathcal{K}(w)$ such that

$$
\begin{aligned}
z_{2}(s)= & \frac{1}{p_{1}} \int_{0}^{s} e^{-p_{2}(s-r)} \int_{0}^{r} \frac{(r-m)^{\varrho-2}}{\Gamma(\varrho-1)} \hat{\vartheta}_{2}(m) \mathrm{d} m \mathrm{~d} r \\
& +\frac{1-e^{-p_{2} s}+\left(p_{2}^{2}-p_{2}\right) s}{p_{1}\left(\tilde{\Omega}_{2}-p_{2} \Delta^{*}\right)}\left[\int_{0}^{1} e^{-p_{2}(1-r)} \int_{0}^{r} \frac{(r-m)^{\varrho-2}}{\Gamma(\varrho-1)} \hat{\vartheta}_{2}(m) \mathrm{d} m \mathrm{~d} r\right. \\
& \left.+\int_{0}^{p} \frac{(p-r)^{\xi-1}}{\Gamma(\xi)} \int_{0}^{r} e^{-p_{2}(r-\tau)} \int_{0}^{\tau} \frac{(\tau-m)^{\varrho-2}}{\Gamma(\varrho-1)} \hat{\vartheta}_{2}(m) \mathrm{d} m \mathrm{~d} \tau \mathrm{d} r\right]
\end{aligned}
$$

for all $s \in[0,1]$. By repeating a process similar to that in the proof of Theorem 10 we conclude that

$$
\left\|z_{1}-z_{2}\right\|=\sup _{s \in[0,1]}\left|z_{1}(s)-z_{2}(s)\right| \leq M\|\delta\| \psi\left(\left\|w-w^{\prime}\right\|\right) \frac{1}{M\|\delta\|}=\psi\left(\left\|w-w^{\prime}\right\|\right),
$$

and so $\mathrm{PH}_{d_{\mathcal{W}}}\left(\mathcal{K}(w), \mathcal{K}\left(w^{\prime}\right)\right) \leq \psi\left(\left\|w-w^{\prime}\right\|\right)$ for all $w, w^{\prime} \in \mathcal{W}$. Furthermore, hypothesis (Hyp14) states that $\mathcal{K}$ has approximate endpoint property. Now by Theorem 4 the operator $\mathcal{K}$ has a unique endpoint, that is, there is $w^{*} \in \mathcal{W}$ such that $\mathcal{K}\left(w^{*}\right)=\left\{w^{*}\right\}$. Hence $w^{*}$ is as a solution of the three-point sequential inclusion BVP (3)-(4).

Now we provide two examples to illustrate our main results.

Example 1 Consider the fractional sequential hybrid differential inclusion

$$
\begin{gathered}
0.08\left({ }^{c} D_{0^{+}}^{2.53}+0.12^{c} D_{0^{+}}^{1.53}\right)\left(\frac{w(s)}{0.0007+\frac{s}{1000}\left(\arcsin w(s)+\sin \left({ }^{R} I_{0^{+}}^{0.04} w(s)\right)\right)}\right) \\
\in\left[0,\left(s+\frac{1}{4}\right) \sin w(s)+\frac{1}{2}\right]
\end{gathered}
$$

with three-point hybrid integro-derivative boundary conditions

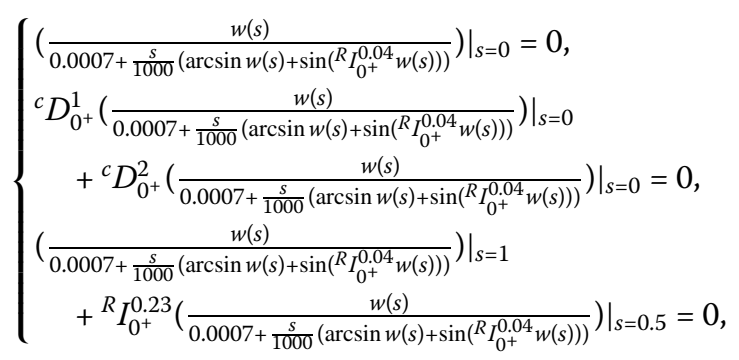

where $s \in[0,1], \varrho=2.53, p_{1}=0.08, p_{2}=0.12, p=0.5, \gamma=0.04$, and $\xi=0.23$. Hence we obtain $\tilde{\Omega}_{1} \simeq 0.1576, \tilde{\Omega}_{2} \simeq 0.008$, and $\Delta^{*} \simeq 0.1656$. Now we define the nonzero continuous map $\zeta:[0,1] \times \mathbb{R} \times \mathbb{R} \rightarrow \mathbb{R} \backslash\{0\}$ by $\zeta\left(s, w_{1}(s), w_{2}(s)\right)=0.0007+\frac{s}{1000}\left(\arcsin w_{1}(s)+\right.$ $\left.\sin \left({ }^{R} I_{0^{+}}^{0.04} w_{2}(s)\right)\right)$ with $\zeta^{*}=\sup _{s \in[0,1]}|\zeta(s, 0,0)|=0.0007$. Let $w, w^{\prime} \in \mathbb{R}$. Then we have

$$
\begin{aligned}
\left|\zeta\left(s, w(s),{ }^{R} I_{0^{+}}^{\gamma} w(s)\right)-\zeta\left(s, w^{\prime}(s),{ }^{R} I_{0^{+}}^{\gamma} w^{\prime}(s)\right)\right| & \leq v(s)\left[1+\frac{s^{\gamma}}{\Gamma(\gamma+1)}\right]\left|w(s)-w^{\prime}(s)\right| \\
& =\frac{s}{1000}\left[1+\frac{s^{0.04}}{\Gamma(1.04)}\right]\left|w(s)-w^{\prime}(s)\right|,
\end{aligned}
$$


where $v(s)=\frac{s}{1000}$ and $v^{*}=\sup _{s \in[0,1]}|v(s)|=\frac{1}{1000}$. Note that the Lipschitz constant of the function $\zeta$ is $v^{*}\left[1+\frac{1}{\Gamma(\gamma+1)}\right]=\frac{1}{1000}\left[1+\frac{1}{\Gamma(1.04)}\right] \simeq 0.002022>0$. Consider the set-valued map $\mathcal{S}:[0,1] \times \mathbb{R} \rightarrow \mathcal{P}(\mathbb{R})$ defined by $\mathcal{S}(s, w(s))=\left[0,\left(s+\frac{1}{4}\right) \sin w(s)+\frac{1}{2}\right]$. Since

$$
|v| \leq \max \left[0,\left(s+\frac{1}{4}\right) \sin w(s)+\frac{1}{2}\right] \leq s+0.75
$$

for each $v \in \mathcal{S}(s, w(s)),\|\mathcal{S}(s, w(s))\|=\sup \{|\hat{\vartheta}|: \hat{\vartheta} \in \mathcal{S}(s, w(s))\} \leq s+0.75$. Now put $\theta(s)=$ $s+0.75$ for $s \in[0,1]$. Then $\|\theta\|_{\mathcal{L}^{1}}=\int_{0}^{1}|\theta(r)| \mathrm{d} r=\int_{0}^{1}(r+0.75) \mathrm{d} r=1.25$ and $M \simeq 171.7012$. Choose $q>0.2654259$. Then

$$
v^{*}\left[1+\frac{1}{\Gamma(\gamma+1)}\right] M\|q\|_{\mathcal{L}^{1}} \simeq(0.002022)(171.7012)(1.25) \simeq 0.433974<\frac{1}{2} .
$$

By Theorem 8 the sequential hybrid inclusion BVP (18)-(19) has a solution.

Example 2 Consider the sequential fractional hybrid differential inclusion

$$
0.08\left({ }^{c} D_{0^{+}}^{2.53}+0.12^{c} D_{0^{+}}^{1.53}\right) w(s) \in\left[0, \frac{5 e^{s}}{7} \frac{|\arctan w(s)|}{1+|\arctan w(s)|}\right]
$$

with three-point integro-derivative boundary conditions

$$
w(0)=0, \quad w^{\prime}(0)+w^{\prime \prime}(0)=0, \quad w(1)+{ }^{R} I_{0^{+}}^{0.23} w(0.5)=0
$$

for $s \in[0,1]$, where ${ }^{c} D_{0^{+}}^{j}$ is the Caputo derivative of order $j \in\{2.53,1.53\}$, and ${ }^{R} I_{0^{+}}^{0.23}$ is the Riemann-Liouville integral. Put $\varrho=2.53, p_{1}=0.08, p_{2}=0.12, p=0.5$, and $\xi=0.23$. Then $\tilde{\Omega}_{1} \simeq 0.1576, \tilde{\Omega}_{2} \simeq 0.008, \Delta^{*} \simeq 0.1656$, and $M \simeq 171.7012$. Define the set-valued map $\mathcal{S}:[0,1] \times \mathcal{W} \rightarrow \mathcal{P}(\mathcal{W})$ by $\mathcal{S}(s, w(s))=\left[0, \frac{5 e^{s}}{7} \frac{|\arctan w(s)|}{1+|\arctan w(s)|}\right]$ for $s \in[0,1]$. Consider the function $\delta \in \mathcal{C}_{\mathbb{R} \geq 0}([0,1])$ defined by $\delta(s)=\frac{5 e^{s}}{7}$ for all $s$ with $\|\delta\|=\frac{5 e}{7} \simeq 1.93571$. Define the nondecreasing nonnegative function $\psi:[0, \infty) \rightarrow[0, \infty)$ by $\psi(s)=\frac{s}{2}$ for all $s>0$. Note that $\psi$ is upper semicontinuous, $\liminf _{s \rightarrow \infty}(s-\psi(s))>0$, and $\psi(s)<s$ for all $s>0$. For every $w, w^{\prime} \in \mathcal{W}$, we have

$$
\begin{aligned}
\mathrm{PH}_{d_{\mathcal{W}}}\left(\mathcal{S}(s, w(s)), \mathcal{S}\left(s, w^{\prime}(s)\right)\right) & \leq \frac{5 e^{s}}{7} \frac{1}{2}\left(\left|w-w^{\prime}\right|\right) \\
& =\frac{5 e^{s}}{7} \psi\left(\left|w-w^{\prime}\right|\right) \leq \delta(s) \psi\left(\left|w-w^{\prime}\right|\right) \frac{1}{M\|\delta\|},
\end{aligned}
$$

where $\frac{1}{M\|\delta\|} \simeq 0.003008$. Consider the operator $\mathcal{K}: \mathcal{W} \rightarrow \mathcal{P}(\mathcal{W})$ defined by

$$
\mathcal{K}(w)=\left\{z \in \mathcal{W} \text { : there is } \hat{\vartheta} \in(\mathcal{S E} \mathcal{L})_{\mathcal{S}, w} \text { such that } z(s)=h(s) \text { for any } s \in[0,1]\right\}
$$

where

$$
\begin{aligned}
h(s)= & \frac{1}{0.08} \int_{0}^{s} e^{-0.12(s-r)} \int_{0}^{r} \frac{(r-m)^{2.53-2}}{\Gamma(2.53-1)} \hat{\vartheta}(m) \mathrm{d} m \mathrm{~d} r \\
& +\frac{1-e^{-0.12 s}+\left((0.12)^{2}-0.12\right) s}{0.08(0.008-(0.12)(0.1656))}
\end{aligned}
$$




$$
\begin{aligned}
& \times\left[\int_{0}^{1} e^{-0.12(1-r)} \int_{0}^{r} \frac{(r-m)^{2.53-2}}{\Gamma(2.53-1)} \hat{\vartheta}(m) \mathrm{d} m \mathrm{~d} r\right. \\
& \left.+\int_{0}^{0.5} \frac{(0.5-r)^{0.23-1}}{\Gamma(0.23)} \int_{0}^{r} e^{-0.12(r-\tau)} \int_{0}^{\tau} \frac{(\tau-m)^{2.53-2}}{\Gamma(2.53-1)} \hat{\vartheta}(m) \mathrm{d} m \mathrm{~d} \tau \mathrm{d} r\right] .
\end{aligned}
$$

Now by Theorem 11 the sequential fractional inclusion (20) has a solution.

\section{Conclusions}

Nowadays we need to study more natural phenomena to obtain more abilities for modeling. The fractional operators were developed over the years, and today their importance has become more and more apparent to researchers. In this way, it is necessary to design different and complicated modelings by utilizing the fractional differential problems. In this work, we review sequential fractional hybrid differential inclusions with three-point integro-derivative boundary value conditions. We employ some analytical tools to study the existence results corresponding to problems (1)-(2) and (3)-(4). We use some notions such as approximate endpoint, $\left(C_{\alpha}\right)$, and the compactness property in this regard. Finally, we provide two examples to illustrate our main results.

\section{Acknowledgements}

The first author was supported by Miandoab Branch of Islamic Azad University. Also, the second and third authors were supported by Azarbaijan Shahid Madani University.

\section{Funding}

Not applicable.

Availability of data and materials

Data sharing not applicable to this paper as no datasets were generated or analyzed during the current study.

Ethics approval and consent to participate

Not applicable.

Competing interests

The authors declare that they have no competing interests.

Consent for publication

Not applicable.

Authors' contributions

The authors declare that the study was realized in collaboration with equal responsibility. All authors read and approved the final manuscript.

\section{Author details}

${ }^{1}$ Department of Mathematics, Miandoab Branch, Islamic Azad University, Miandoab, Iran. ${ }^{2}$ Institute of Research and Development, Duy Tan University, Da Nang 550000, Vietnam. ${ }^{3}$ Faculty of Natural Sciences, Duy Tan University, Da Nang 550000, Vietnam. ${ }^{4}$ Department of Medical Research, China Medical University Hospital, China Medical University, Taichung, Taiwan. ${ }^{5}$ Department of Mathematics, Azarbaijan Shahid Madani University, Tabriz, Iran. ${ }^{6}$ Department of Mathematics, Cankaya University, Ogretmenler Cad. 1406530 Balgat, Ankara, Turkey. ${ }^{7}$ Institute of Space Sciences, Bucharest, Romania.

\section{Publisher's Note}

Springer Nature remains neutral with regard to jurisdictional claims in published maps and institutional affiliations.

Received: 12 May 2020 Accepted: 22 July 2020 Published online: 28 July 2020

References

1. Alizadeh, S., Baleanu, D., Rezapour, S.: Analyzing transient response of the parallel RCL circuit by using the Caputo-Fabrizio fractional derivative. Adv. Differ. Equ. 2020, 55 (2020). https://doi.org/10.1186/s13662-020-2527-0

2. Baleanu, D., Jajarmi, A., Mohammadi, H., Rezapour, S.: Analysis of the human liver model with Caputo-Fabrizio fractional derivative. Chaos Solitons Fractals 134, 7 (2020)

3. Baleanu, D., Mohammadi, H., Rezapour, S.: Analysis of the model of HIV-1 infection of CD4 ${ }^{+}$T-cell with a new approach of fractional derivative. Adv. Differ. Equ. 2020, 71 (2020). https://doi.org/10.1186/s13662-020-02544-w 
4. Baleanu, D., Mohammadi, H., Rezapour, S.: A mathematical theoretical study of a particular system of Caputo-Fabrizio fractional differential equations for the rubella disease model. Adv. Differ. Equ. 2020, 184 (2020). https://doi.org/10.1186/s13662-020-02614-Z

5. Zhou, H., Alzabut, J., Rezapour, S., Samei, M.E.: Uniform persistence and almost periodic solutions of a non-autonomous patch occupancy model. Adv. Differ. Equ. 2020, 143 (2020). https://doi.org/10.1186/s13662-020-02603-2

6. Baleanu, D., Mohammadi, H., Rezapour, S.: A fractional differential equation model for the COVID-19 transmission by using the Caputo-Fabrizio derivative. Adv. Differ. Equ. 2020, 299 (2020). https://doi.org/10.1186/s13662-020-02762-2

7. Baleanu, D., Etemad, S., Rezapour, S.: A hybrid Caputo fractional modeling for thermostat with hybrid boundary value conditions. Bound. Value Probl. 2020, 64 (2020). https://doi.org/10.1186/s13661-020-01361-0

8. Baleanu, D., Ghafarnezhad, K., Rezapour, S.: On a three steps crisis integro-differential equation. Adv. Differ. Equ. 2019, $153(2019)$

9. Aydogan, M., Baleanu, D., Mousalou, A., Rezapour, S.: On high order fractional integro-differential equations including the Caputo-Fabrizio derivative. Bound. Value Probl. 2018, 90 (2018). https://doi.org/10.1186/s13661-018-1008-9

10. Aydogan, M., Baleanu, D., Mousalou, A., Rezapour, S.: On approximate solutions for two higher-order Caputo-Fabrizio fractional integro-differential equations. Adv. Differ. Equ. 2017, 221 (2017). https://doi.org/10.1186/s13662-017-1258-3

11. Baleanu, D., Mohammadi, H., Rezapour, S.: On a nonlinear fractional differential equation on partially ordered metric spaces. Adv. Differ. Equ. 2013, 83 (2013). https://doi.org/10.1186/1687-1847-2013-83

12. Baleanu, D., Rezapour, S., Mohammadi, H.: Some existence results on nonlinear fractional differential equations. Philos. Trans. R. Soc. A 371, 20120144 (2013). https://doi.org/10.1098/rsta.2012.0144

13. Baleanu, D., Mousalou, A., Rezapour, S.: A new method for investigating approximate solutions of some fractional integro-differential equations involving the Caputo-Fabrizio derivative. Adv. Differ. Equ. 2017(1), 51 (2017) https://doi.org/10.1186/s13662-017-1088-3

14. Baleanu, D., Mousalou, A., Rezapour, S.: The extended fractional Caputo-Fabrizio derivative of order $0 \leq \sigma<1$ on c $[0,1]$ and the existence of solutions for two higher-order series-type differential equations. Adv. Differ. Equ. 2018(1), 255 (2018). https://doi.org/10.1186/s13662-018-1696-6

15. Baleanu, D., Mousalou, A., Rezapour, S.: On the existence of solutions for some infinite coefficient-symmetric Caputo-Fabrizio fractional integro-differential equations. Bound. Value Probl. 2017(1), 145 (2017). https://doi.org/10.1186/s13661-017-0867-9

16. Etemad, S., Pourrazi, S., Rezapour, S.: On a hybrid inclusion problem via hybrid boundary value conditions. Adv. Differ. Equ. 2020, 302 (2020). https://doi.org/10.1186/s13662-020-02764-0

17. Charandabi, Z.Z., Rezapour, S., Ettefagh, M.: On a fractional hybrid version of the Sturm-Liouville equation. Adv. Differ Equ. 2020, 301 (2020). https://doi.org/10.1186/s13662-020-02765-z

18. Etemad, S., Rezapour, S., Samei, M.E.: $\alpha$ - $\psi$-contractions and solutions of a $q$-fractional differential inclusion with three-point boundary value conditions via computational results. Adv. Differ. Equ. 2020, 218 (2020). https://doi.org/10.1186/s13662-020-02679-w

19. Etemad, S., Rezapour, S., Sakar, F.M.: On a fractional Caputo-Hadamard problem with boundary value conditions via different orders of the Hadamard fractional operators. Adv. Differ. Equ. 2020, 272 (2020). https://doi.org/10.1186/s13662-020-02741-7

20. Etemad, S., Rezapour, S.: On the existence of solutions for fractional boundary value problems on the Ethane graph Adv. Differ. Equ. 2020, 276 (2020). https://doi.org/10.1186/s13662-020-02736-4

21. Baleanu, D., Rezapour, S., Saberpour, Z.: On fractional integro-differential inclusions via the extended fractional Caputo-Fabrizio derivation. Bound. Value Probl. 2019, 79 (2019). https://doi.org/10.1186/s13661-019-1194-0

22. Talaee, M., Shabibi, M., Gilani, A., Rezapour, S.: On the existence of solutions for a pointwise defined multi-singular integro-differential equation with integral boundary condition. Adv. Differ. Equ. 2020, 41 (2020). https://doi.org/10.1186/s13662-020-2517-2

23. Miller, K.S., Ross, B.: An Introduction to Fractional Calculus and Fractional Differential Equations. Wiley, New York (1993)

24. Wei, Z., Dong, W.: Periodic boundary value problems for Riemann-Liouville sequential fractional differential equations. Electron. J. Qual. Theory Differ. Equ. 2011, 87 (2011)

25. Wei, Z., Li, Q., Che, J.: Initial value problems for fractional differential equations involving Riemann-Liouville sequential fractional derivative. J. Math. Anal. Appl. 367, 260-272 (2010)

26. Ahmad, B., Nieto, J.J.: Boundary value problems for a class of sequential integro-differential equations of fractional order. J. Funct. Spaces 2013, 149659 (2013). https://doi.org/10.1155/2013/149659

27. Ahmad, B., Ntouyas, S.K.: On higher-order sequential fractional differential inclusions with nonlocal three-point boundary conditions. Abstr. Appl. Anal. 2014, 659405 (2014). https://doi.org/10.1155/2014/659405

28. Aljoudi, S., Ahmad, B., Nieto, J.J., Alsaedi, A.: A coupled system of Hadamard type sequential fractional differential equations with coupled strip conditions. Chaos Solitons Fractals 91, 39-46 (2016). https://doi.org/10.1016/j.chaos.2016.05.005

29. Bai, C.: Impulsive periodic boundary value problems for fractional differential equation involving Riemann-Liouville sequential fractional derivative. J. Math. Anal. Appl. 384, 211-231 (2011)

30. Klimek, M.: Sequential fractional differential equations with Hadamard derivative. Commun. Nonlinear Sci. Numer. Simul. 16, 4689-4697 (2011)

31. Alsaedi, A., Ntouyas, S.K., Agarwal, R.P., Ahamad, B.: On Caputo type sequential fractional differential equations with nonlocal integral boundary conditions. Adv. Differ. Equ. 2015, 33 (2015). https://doi.org/10.1186/s13662-015-0379-9

32. Hilal, K., Kajouni, A.: Boundary value problems for hybrid differential equations with fractional order. Adv. Differ. Equ. 2015, $183(2015)$

33. Samei, M.E., Hedayati, V., Rezapour, S.: Existence results for a fraction hybrid differential inclusion with Caputo-Hadamard type fractional derivative. Adv. Differ. Equ. 2019, 163 (2019). https://doi.org/10.1186/s13662-019-2090-8

34. Sun, S., Zhao, Y., Han, Z., Li, Y.: The existence of solutions for boundary value problem of fractional hybrid differential equations. Commun. Nonlinear Sci. Numer. Simul. 17, 4961-4967 (2012) 
35. Dhage, B.C., Lakshmikantham, V.: Basic results on hybrid differential equation. Nonlinear Anal. Hybrid Syst. 4, 414-424 (2010)

36. Zhao, Y., Sun, S., Han, Z., Li, Q.: Theory of fractional hybrid differential equations. Comput. Math. Appl. 62(3), 1312-1324 (2011). https://doi.org/10.1016/j.camwa.2011.03.041

37. Ahmad, B., Ntouyas, S.K., Tariboon, J.: On hybrid Caputo fractional integro-differential inclusions with nonlocal conditions. J. Nonlinear Sci. Appl. 9(6), 4235-4246 (2016). https://doi.org/10.22436/jnsa.009.06.65

38. Baleanu, D., Hedayati, V., Rezapour, S., Al Qurashi, M.M.: On two fractional differential inclusions. SpringerPlus 5(1), 882 (2016). https://doi.org/10.1186/s40064-016-2564-z

39. Baleanu, D., Etemad, S., Pourrazi, S., Rezapour, S.: On the new fractional hybrid boundary value problems with three-point integral hybrid conditions. Adv. Differ. Equ. 2019, 473 (2019)

40. Podlubny, I.: Fractional Differential Equations. Academic Press, San Diego (1999)

41. Samko, S.G., Kilbas, A.A., Marichev, O.I.: Fractional Integrals and Derivatives: Theory and Applications. Gordon \& Breach, Philadelphia (1993)

42. Deimling, K.: Multi-Valued Differential Equations. de Gruyter, Berlin (1992)

43. Aubin, J., Cellina, A.: Differential Inclusions: Set-Valued Maps and Viability Theory. Springer, Berlin (1984) https://doi.org/10.1007/978-3-642-69512-4

44. Samet, B., Vetro, C., Vetro, P.: Fixed point theorems for $\alpha-\psi$-contractive type mappings. Nonlinear Anal. 75, 2154-2165 (2012)

45. Mohammadi, B., Rezapour, S., Shahzad, N.: Some results on fixed points of $\alpha$ - $\psi$-Ciric generalized multifunctions. Fixed Point Theory Appl. 2013, 24 (2013). https://doi.org/10.1186/1687-1812-2013-24

46. Amini-Harandi, A.: On Caputo type sequential fractional differential equations with nonlocal integral boundary conditions. Nonlinear Anal. 72, 132-134 (2010)

47. Lasota, A., Opial, Z:: An application of the Kakutani-Ky Fan theorem in the theory of ordinary differential equations. Bull. Acad. Pol. Sci., Sér. Sci. Math. Astron. Phys. 13, 781-786 (1965)

48. Dhage, B.C.: Existence results for neutral functional differential inclusions in Banach algebras. Nonlinear Anal. 64 1290-1306 (2006)

\section{Submit your manuscript to a SpringerOpen ${ }^{\circ}$ journal and benefit from:}

- Convenient online submission

- Rigorous peer review

Open access: articles freely available online

- High visibility within the field

- Retaining the copyright to your article

Submit your next manuscript at $>$ springeropen.com 